$\mathrm{SPhT} / 00-145$

NBI-HE-00-39

\title{
Generalized Lorentzian Triangulations and the Calogero Hamiltonian
}

\author{
P. Di Francescol, E. Guitter日 \\ CEA-Saclay, Service de Physique Théorique, \\ F-91191 Gif sur Yvette Cedex, France \\ C. Kristjansen 3 \\ The Niels Bohr Institute, Blegdamsvej 17, \\ DK-2100 Copenhagen Ø, Denmark
}

\begin{abstract}
We introduce and solve a generalized model of $1+1 \mathrm{D}$ Lorentzian triangulations in which a certain subclass of outgrowths is allowed, the occurrence of these being governed by a coupling constant $\beta$. Combining transfer matrix-, saddle point- and path integral techniques we show that for $\beta<1$ it is possible to take a continuum limit in which the model is described by a $1 \mathrm{D}$ quantum Calogero Hamiltonian. The coupling constant $\beta$ survives the continuum limit and appears as a parameter of the Calogero potential.
\end{abstract}

$10 / 00$

$\begin{array}{ll}1 & \text { philippe@spht.saclay.cea.fr } \\ 2 & \text { guitter@spht.saclay.cea.fr } \\ 3 & \text { kristjan@nbi.dk, supported by the Carlsberg Foundation }\end{array}$ 


\section{Introduction}

Random triangulations are interesting both from a field theoretic and from a statistical mechanical point of view (for a review, see for instance [1-2]). In particular, the socalled dynamical triangulations, also denoted in the following as Euclidean triangulations, provide us with a consistent lattice regularized version of 2D Euclidean quantum gravity, which in its scaling limit reproduces the continuum Liouville field theory. Moreover, the introduction of statistical mechanical degrees of freedom on dynamical triangulations also led to the discovery of new universality classes describing critical phenomena on fluctuating surfaces.

Beside dynamical triangulations, another type of random triangulations has recently been introduced, known as 1+1D Lorentzian triangulations [3]. What characterizes a Lorentzian triangulation is that it can be decomposed into slices by cutting along horizontal lines, as shown in Fig. 1. Each slice is made of an arbitrary sequence of triangles pointing up or down at random. Each triangle has two "time-like" edges within the slice and one "space-like" edge shared with a triangle in a neighboring slice.

(a)

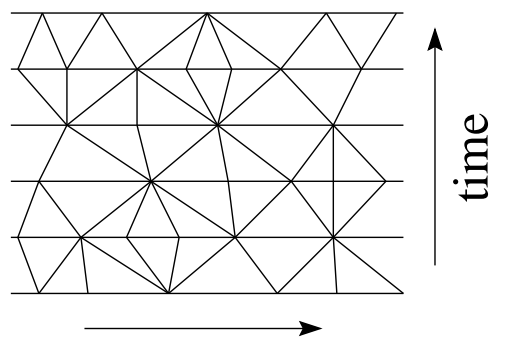

space (b)

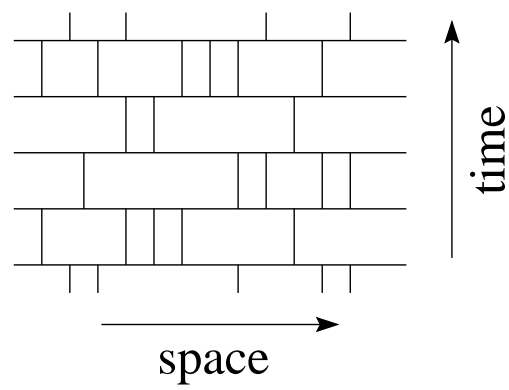

Fig. 1: A Lorentzian triangulation (a) and its dual (b).

Lorentzian triangulations were invented with the aim of constructing a model of quantum gravity where causality was built in at a fundamental level. The resulting gravity model was denoted as Lorentzian gravity [3]. This model has a well defined scaling limit which is different from that of Euclidean gravity. At present there does not exist any continuum formulation à la Liouville with such a causal structure. We note, however, that a seed of such a formulation might be found in reference [4. Lorentzian triangulations are also interesting in their own right as a new statistical model, allowing for the definition of a new class of lattice models. Lorentzian triangulations lie somewhere between regular lattices with defects and completely random lattices (i.e. dynamical triangulations) and it is possible that models based on this type of lattices will reveal yet other universality classes. 


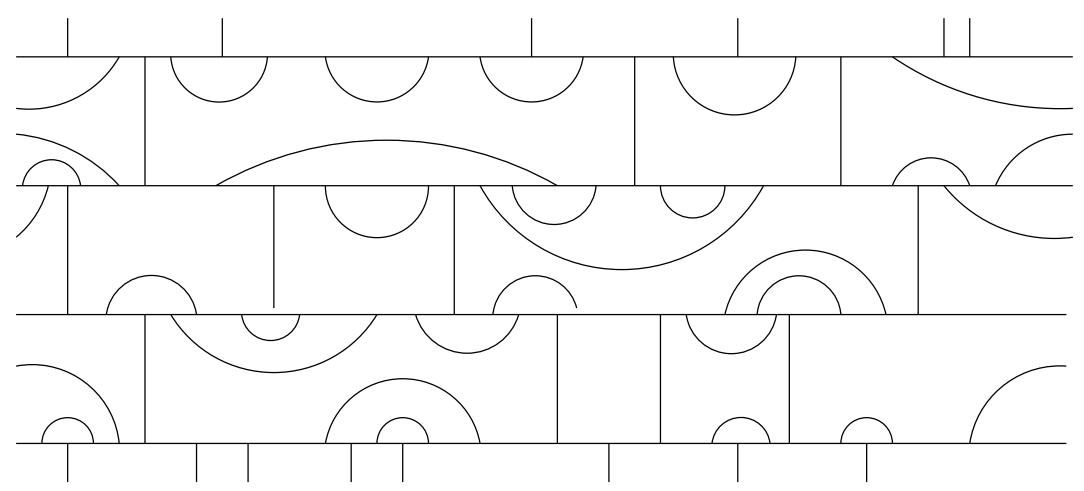

Fig. 2: A generalized Lorentzian triangulation in the dual picture.

In this spirit we will study in the present paper a generalized model of Lorentzian triangulations where the time slice structure is preserved but where some outgrowths within the time slices are allowed.

Such a generalized Lorentzian triangulation is displayed in Fig.2 in the dual picture and is obtained by decorating an ordinary Lorentzian triangulation such as that of Fig.1 (b) by adding arbitrary space-like "arches" connecting two points of the same time line. These arches do not intersect each other nor the vertical straight lines. For later convenience, the boundary condition imposed on the arch configurations is such that any number of arches can escape from the right hand side of a given slice and reappear on the left hand side, see Fig. 2. An elementary arch, in the language of triangulations, corresponds to a pair of connected triangles that do not propagate in time, as opposed to pairs of triangles dual to a vertical edge. In the terminology of quantum gravity such an object constitutes a particular type of (small) "baby-universe". Keeping to the statistical mechanical language we will simply refer to such decorations as "outgrowths". Similarly, more involved arch configurations correspond to more involved outgrowths each living in a single time-slice. Clearly, the class of outgrowths we allow for constitutes only a small subset of the babyuniverses present in the dynamically triangulated or Euclidean quantum gravity model. In order to get all baby-universes appearing in Euclidean gravity one would have to allow for loops connected in all possible ways to the already existing elements of the lattice via three-valent lattices. Such a model has been studied in ref. [5]. It corresponds to the fully packed phase of the so-called $O(1)$ model on a random lattice. Note finally that the outgrowths we consider would not suffice to generate surfaces of arbitrary topology. The surfaces we consider have genus zero or one depending on boundary conditions.

Our motivation for studying the particular type of lattices depicted in Fig. 2 is twofold. First, in the context of $2 \mathrm{D}$ quantum gravity, it has been shown that one can view 
Lorentzian quantum gravity as a renormalized version of Euclidean quantum gravity [6]. Obviously, in going from Euclidean to Lorentzian triangulations baby-universes must be integrated over. A key point in the above renormalization procedure consists in a nonanalytical redefinition of the "boundary cosmological constant" which is the parameter coupled to the length of the spatial slices, i.e. the length of the horizontal lines in Fig. 1 or Fig. 2. In our model all outgrowths lie along the horizontal lines and one of our aims is to investigate whether the redefinition of the boundary cosmological constant can be due to these decorations alone.

Secondly, from the statistical mechanical point of view, lattices of the type depicted in Fig. 2 are interesting because the system of arch configurations is known to be critical [7] and by defining this system on Lorentzian triangulations one might be able to change the universality class of the geometrical system. So far one has not been able to study analytically the behavior of Lorentzian triangulations when critical matter fields are introduced. One has studied numerically systems consisting of one to eight Ising spins on Lorentzian triangulations [8]. These studies show that the interaction between matter and geometry is much weaker than for the models based on dynamical triangulations. Analytical investigations have been carried out for Lorentzian triangulations equipped with various dimer fields and for the case where higher curvature interactions are present [9]. These models all turned out to belong to the same universality class as pure Lorentzian triangulations. Remarkably, the concept of integrability generalized to non-regular (Lorentzian) lattices played a crucial role in their exact solutions and led to the hope of extending the standard techniques of integrable lattice models to models on fluctuating lattices.

The paper is organized as follows. In Sect. 2 we describe in more detail the generalized Lorentzian triangulations that we are going to study and write down their transfer matrix description. Next, in Sect. 3 we reformulate the model using a Schwinger-type integral representation leading to a continuous transfer matrix. The Schwinger language provides us with an alternative way of obtaining the exact solution in the case of pure Lorentzian triangulations. In Sect. 4 we consider the case where arches or outgrowths are present and show the existence of three different regimes of the model according to the value of a parameter $\beta$ governing the density of outgrowths. In the interesting case of low density of outgrowths $(\beta<1)$, we define in Sect. 5 a sensible scaling limit in which our model gets identified with a well-known quantum mechanical system, namely that governed by onedimensional Calogero Hamiltonian. Using this equivalence, we compute several interesting thermodynamic quantities. Finally, we discuss in Sect. 6 various aspects of our results. 


\section{The model}

Our generalized model is based on triangular lattices which in the dual picture look as in Fig. 2. In addition to a Boltzmann weight $g$ per triangle, we introduce a weight $h$ for each triangle which in the dual language is part of a vertical edge and a weight $\theta$ for each triangle which in the dual language is part of an arch, see Fig. 3. We also introduce the parameter

$$
\beta=\frac{\theta}{h},
$$

governing the density of arch decorations.
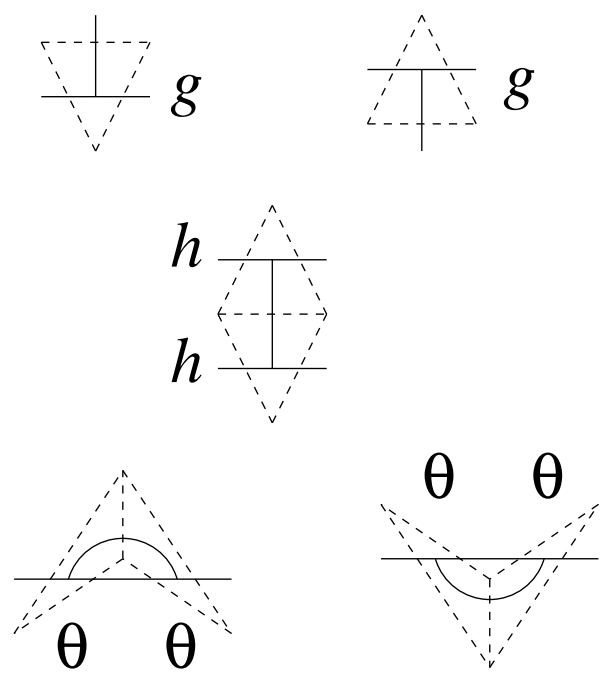

Fig. 3: The weights associated with the different elements of a generalized Lorentzian triangulation.

The partition function of our model over a time lapse $t$ is obtained by summing over all such generalized triangulations with exactly $t$ time slices and weighted as just explained.

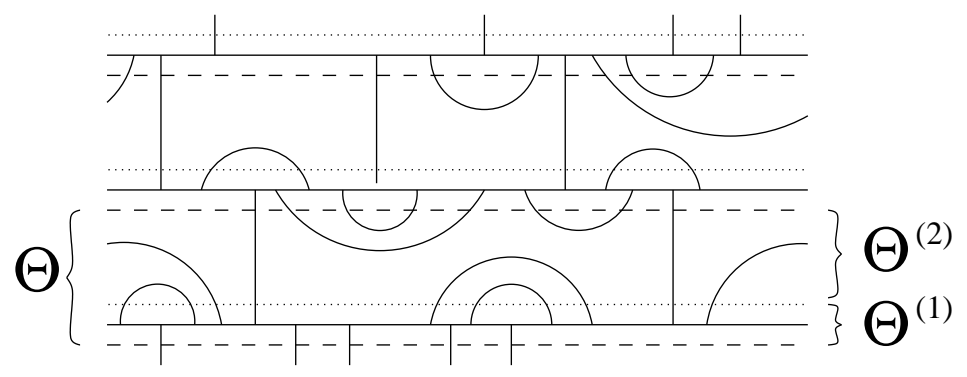

Fig. 4: The transfer matrix $\Theta$ is decomposed into two parts: the matrix $\Theta^{(1)}$ of the pure case, and the matrix $\Theta^{(2)}$ implementing the upper and lower arch decorations. 
As in the case of pure Lorentzian triangulations, we may describe our model using a transfer matrix approach. The transfer matrix describes the effect of adding one time-slice to our lattice. In the present case it is convenient to view the addition of one slice of the lattice as two consecutive steps. We first apply the transfer matrix of pure Lorentzian triangulations $\Theta^{(1)}$ which corresponds to adding the portion of lattice below the dotted line and above the dashed line in Fig. 4, including a line of constant time. The transfer matrix element $\Theta_{i j}^{(1)}$ is indexed by two non-negative integers $i$ and $j$ which denote the number of half-edges going into the time line and that coming out of the line respectively and counts the (weighted) arrangements of these half-edges along the time line. We then complete the slice addition by applying a transfer matrix $\Theta^{(2)}$ which corresponds to adding the portion of lattice above the dotted line and below the dashed one in Fig. 4 and accounts for the pairing of half-edges into arches or into vertical edges. The transfer matrix element $\Theta_{i j}^{(2)}$ is also indexed by the numbers of in and out-coming half edges. The total transfer matrix $\Theta$ can therefore be written as a product of the two above transfer matrices

$$
\Theta_{i j}=\sum_{k} \Theta_{i k}^{(1)} \Theta_{k j}^{(2)}
$$

In the case $\theta=0, h=1$ of pure Lorentzian triangulations, the matrix $\Theta^{(2)}$ reduces to the identity. The new ingredient is therefore entirely contained in the definition of $\Theta^{(2)}$.

For convenience we will work mainly in the language of generating functions, by defining

$$
\Theta(x, y)=\sum_{i, j=0}^{\infty} \Theta_{i j} x^{i} y^{j}
$$

and similarly for $\Theta^{(1)}$ and $\Theta^{(2)}$. The composition law 2.2 then reads

$$
\Theta(x, y)=\oint_{\mathcal{C}} \frac{d \omega}{2 \pi i \omega} \Theta^{(1)}\left(x, \frac{1}{\omega}\right) \Theta^{(2)}(\omega, y),
$$

with the contour $\mathcal{C}$ encircling the origin. The generating functions can be easily derived, with the result

$$
\begin{aligned}
& \Theta^{(1)}(x, y)=\frac{1}{1-g x-g y} \\
& \Theta^{(2)}(x, y)=\frac{C\left(\theta^{2} x^{2}\right)}{1-\theta^{2} x^{2} C^{2}\left(\theta^{2} x^{2}\right)} \frac{C\left(\theta^{2} y^{2}\right)}{1-\theta^{2} y^{2} C^{2}\left(\theta^{2} y^{2}\right)} \frac{1}{1-h^{2} x y C\left(\theta^{2} x^{2}\right) C\left(\theta^{2} y^{2}\right)}
\end{aligned}
$$


where $C(z)$ is the generating function for Catalan numbers

$$
C(z)=\frac{1-\sqrt{1-4 z}}{2 z}
$$

The result for $\Theta^{(1)}$ is the direct consequence of the matrix element $\Theta_{i j}^{(1)}$ taking the value $\left(\begin{array}{c}i+j \\ i\end{array}\right) g^{i+j}$, where we choose for convenience to include the weight $g$ per triangle in $\Theta^{(1)}$. The derivation of $\Theta^{(2)}$ is slightly more involved: we attach to each vertical edge the upper and lower arch configurations sitting immediately to its right (see Fig.4). This results in an effective weight $h^{2} x y C\left(\theta^{2} x^{2}\right) C\left(\theta^{2} y^{2}\right)$ per vertical edge. Indeed, $C(z)$ is the generating function for arbitrary arch configurations with a weight $z$ per arch. Summing over the number of vertical edges leads to the third factor in the second line of (2.5). The first two factors come from our particular choice of boundary conditions. Each is obtained by summing over the number of escaping arches each effectively weighted by a factor $\theta^{2} z^{2} C^{2}\left(\theta^{2} z^{2}\right)$, with $z=x$ resp. $y$ for upper, resp. lower escaping arches. We remark that as opposed to what is the case for $\Theta^{(1)}$ and $\Theta^{(2)}$, the transfer matrix $\Theta$ is not symmetric with respect to $x$ and $y$.

Among other quantities of interest, we will compute the $t$-step partition function with periodic boundary conditions in the time direction

$$
Z(t)=\operatorname{Tr}\left(\Theta^{t}\right)
$$

We will also calculate correlation functions for the total number $N(s)$ of triangles in the slice at time $s$, with the same $t$-step periodic boundary conditions.

Apart from these we will be interested in calculating the $t$-step partition function with open boundaries in the $t$-direction $Z(i, j, t)$

$$
Z(i, j, t)=\left(\left(\Theta^{(1)} \Theta^{(2)}\right)^{t} \Theta^{(1)}\right)_{i j}
$$

4 This asymmetry of $\Theta(x, y)$ can be cured by, instead of defining $\Theta$ by (2.2), setting $\Theta=\left(\Theta^{(1)}\right)^{1 / 2} \Theta^{(2)}\left(\Theta^{(1)}\right)^{1 / 2}$. Since the transfer matrix of the pure model has been explicitly diagonalized [9] it is straightforward to write down an expression for $\left(\Theta^{(1)}\right)^{1 / 2}$. However, the full transfer matrix $\Theta_{i j}$ resulting from this definition has no longer a clear geometrical interpretation as being associated with $i$ incoming edges and $j$ outgoing ones. In appendix $\mathrm{A}$ we show how to calculate $\Theta=\left(\Theta^{(1)}\right)^{1 / 2} \Theta^{(2)}\left(\Theta^{(1)}\right)^{1 / 2}$. Here we shall take a line of action which does not require the knowledge of the exact transfer matrix. 
or rather its associated generating function $Z(x, y, t)$. With a slight abuse of language we will also refer to $Z(x, y, t)$ or $Z(i, j, t)$ as the loop-loop correlator. Notice that $Z(i, j, t)$ is not simply the $t$-th power of the transfer matrix. It is, however, to our opinion the most natural definition of a $t$-step partition function with open boundaries in the $t$-direction. It describes triangulations for which the boundary conditions in the $t$-direction are as depicted in Fig. 2. There are $i$ incoming vertical edges and $j$ outgoing ones and no arches are allowed either between incoming or outgoing edges.

For pure Lorentzian triangulations, two different strategies were used for calculating objects as given above. One strategy consists in first determining $\Theta^{t}(x, y)$ by writing down and solving a recursion relation in $t$ for this quantity [3]. The other one consists in explicitly diagonalizing the transfer matrix $\Theta_{i j}$ [9]. Neither method appears to be tractable for the present problem with general $\theta$ and $h$. We will resort to yet another approach using an integral representation of the transfer matrix.

\section{Schwinger representation}

\subsection{Transfer matrix}

In this section we will reformulate the discrete transfer matrix $\Theta$ with indices $i, j=$ $0,1,2, \ldots$ as a continuous transfer kernel by means of an integral representation, using continuous Schwinger parameters $\alpha, \alpha^{\prime} \in[0, \infty)$. This representation is inspired by the calculation in ref. [5] of the "watermelon" correlation function of the $O(n)$ model coupled to $2 \mathrm{D}$ Euclidean gravity. For definiteness, let us consider the partition function $Z(t)(2.7)$. From (2.4) it follows that

$$
Z(t)=\prod_{s=1}^{t}\left(\oint_{\mathcal{C}} \frac{d w_{s}}{2 i \pi w_{s}} \frac{d z_{s}}{2 i \pi z_{s}}\right) \prod_{r=1}^{t} \Theta^{(1)}\left(\frac{1}{w_{r-1}}, \frac{1}{z_{r}}\right) \Theta^{(2)}\left(z_{r}, w_{r}\right)
$$

where the contour is a small circle around the origin and where we have imposed cyclic boundary conditions on the $w$ 's, namely $w_{0}=w_{t}$.

Whereas in the pure case the integrand in (3.1) would have singularities only in the form of poles, in the present case the integrand in addition has singularities in the form of cuts. We can, however, transform the cuts into poles by the following change of variables

$$
\mu_{r}=\theta w_{r} C\left(\theta^{2} w_{r}^{2}\right), \quad \rho_{r}=\theta z_{r} C\left(\theta^{2} z_{r}^{2}\right)
$$


This change of variables maps the circle $\mathcal{C}$ into a closed ellipse-like curve $\mathcal{E}$ still encircling the origin. Using the quadratic equation satisfied by the Catalan generating function

$$
x(C(x))^{2}=C(x)-1
$$

the relation (3.2) is easily inverted into

$$
w_{r}=\frac{1}{\theta\left(\mu_{r}+\frac{1}{\mu_{r}}\right)}, \quad z_{r}=\frac{1}{\theta\left(\rho_{r}+\frac{1}{\rho_{r}}\right)}
$$

From there it follows that

$$
\frac{d w_{r}}{w_{r}^{2}}=\frac{\theta\left(1-\mu_{r}^{2}\right) d \mu_{r}}{\mu_{r}^{2}}
$$

and a similar relation holds between $d z_{r}$ and $d \rho_{r}$. Inserting (3.2) and (3.5) in (3.1) we get

$$
Z(t)=\prod_{s=1}^{t}\left(\oint_{\mathcal{E}} \frac{d \mu_{s}}{2 i \pi \mu_{s}} \frac{d \rho_{s}}{2 i \pi \rho_{s}}\right) \prod_{r=1}^{t} \frac{1}{1-\frac{\rho_{r} \mu_{r}}{\beta^{2}}} \frac{1}{1-g \theta\left(\rho_{r}+\frac{1}{\rho_{r}}+\mu_{r-1}+\frac{1}{\mu_{r-1}}\right)},
$$

where $\beta=\theta / h$ as in (2.1). We may next introduce a Schwinger representation for the second factor in each of the products in the integrand. This gives

$$
Z(t)=\prod_{s=1}^{t}\left(\oint_{\mathcal{E}} \frac{d \mu_{s}}{2 i \pi \mu_{s}} \frac{d \rho_{s}}{2 i \pi \rho_{s}} \int_{0}^{\infty} d \alpha_{s} e^{-\alpha_{s}}\right) \prod_{r=1}^{t} \frac{1}{1-\frac{\rho_{r} \mu_{r}}{\beta^{2}}} e^{g \theta \alpha_{r}\left(\rho_{r}+\frac{1}{\rho_{r}}+\mu_{r-1}+\frac{1}{\mu_{r-1}}\right)}
$$

The integrals over the $\rho_{r}$ may now be performed, by noticing that each of them simply picks the residue of the integrand at $\rho_{r}=0$ This gives

$$
Z(t)=\prod_{s=1}^{t}\left(\oint_{\mathcal{E}} \frac{d \mu_{s}}{2 i \pi \mu_{s}} \int_{0}^{\infty} d \alpha_{s} e^{-\alpha_{s}}\right) \prod_{r=1}^{t} \sum_{k \geq 0} I_{k}\left(2 g \theta \alpha_{r}\right)\left(\frac{\mu_{r}}{\beta^{2}}\right)^{k} e^{g \theta \alpha_{r+1}\left(\mu_{r}+\frac{1}{\mu_{r}}\right)}
$$

where the $I_{k}$ 's are the modified Bessel functions of the first kind, i.e. $I_{k}(2 x)=$ $\sum_{p \geq 0} x^{2 p+k} /(p !(p+k) !)$ and where we have imposed periodic boundary conditions on the $\alpha$ 's, i.e. $\alpha_{t+1}=\alpha_{1}$.

Similarly, to carry out the integral over $\mu_{r}$ we simply have to pick the residue of the integrand at $\mu_{r}=0$ and the expression finally reduces to

$$
Z(t)=\prod_{s=1}^{t}\left(\int_{0}^{\infty} d \alpha_{s} e^{-\alpha_{s}}\right) \prod_{r=1}^{t} \phi_{\beta}\left(g \theta \alpha_{r}, g \theta \alpha_{r+1}\right)
$$


where we have defined the transition function

$$
\phi_{\beta}(x, y)=\sum_{k \geq 0} I_{k}(2 x) I_{k}(2 y) / \beta^{2 k} .
$$

Note that as expected the partition function depends on $g, h$ and $\theta$ only via $g \theta$ and $\beta=\theta / h$.

Eqn. (3.9) leads to the definition of a continuous symmetric transfer kernel

$$
G_{\beta, g \theta}\left(\alpha, \alpha^{\prime}\right)=e^{-\frac{\alpha+\alpha^{\prime}}{2}} \phi_{\beta}\left(g \theta \alpha, g \theta \alpha^{\prime}\right)
$$

to be integrated with the flat measure on the parameters $\alpha \geq 0$. To make contact with the original discrete formulation of the problem, let us show how to obtain statistical properties involving the observable $N(s)=i+j$ counting the total number of triangles in the slice at time $s$ ( $i$ and $j$ denote respectively the number of in- and out-coming half edges at the time-line $s$ ), from the statistical properties of the variable $\alpha_{s}$. Let us define a more general local observable $\Sigma_{s}(z)$ through

$$
\begin{aligned}
\sigma_{n}(s) & =N(s)(N(s)-1) \ldots(N(s)-n+1), \\
\Sigma_{s}(z) & =\sum_{n=0}^{\infty} \sigma_{n}(s) \frac{z^{n}}{n !} .
\end{aligned}
$$

The correlations of $\Sigma$ at various times $s_{1}, \ldots, s_{k}$ read simply

$$
\left\langle\prod_{m=1}^{k} \Sigma_{s_{m}}\left(z_{m}\right)\right\rangle_{\Theta}=\left\langle\prod_{m=1}^{k} \frac{e^{\frac{\alpha_{s_{m}} z_{m}}{1+z_{m}}}}{1+z_{m}}\right\rangle_{G}
$$

where the subscripts $\Theta, G$ refer to the framework (discrete, continuum) in which the correlation is evaluated. This is readily proved by noticing that the desired correlator corresponds to substitutions $g \rightarrow g\left(1+z_{m}\right)$ in the formula for the partition function (3.9) within the slice $s_{m}$. The latter can then be absorbed into a change of variables $\alpha_{s_{m}} \rightarrow \alpha_{s_{m}} /\left(1+z_{m}\right)$. This will be extensively used in Sect. 5 below.

\subsection{Example 1: pure Lorentzian triangulations revisited}

As a preliminary exercise, it is instructive to re-derive the exact results of pure Lorentzian triangulations in this new continuum language. Let us diagonalize the continuum transfer matrix of pure Lorentzian triangulations (with $\theta=0$ and $h=1$ )

$$
G^{(0)}\left(\alpha, \alpha^{\prime}\right)=\lim _{\beta \rightarrow 0} G_{\beta, g \beta}\left(\alpha, \alpha^{\prime}\right)=e^{-\frac{\alpha+\alpha^{\prime}}{2}} I_{0}\left(2 g \sqrt{\alpha \alpha^{\prime}}\right)
$$


where we have used that $I_{k}(2 g \beta x) / \beta^{k} \rightarrow(g x)^{k} / k !$ in (3.10). It turns out that the eigenfunctions of $G^{(0)}$ are simply related to the Laguerre polynomials $L_{n}(x)$, orthogonal wrt. the measure $e^{-x} d x$ over the positive reals. These polynomials read

$$
L_{n}(x)=\sum_{m=0}^{n}\left(\begin{array}{l}
n \\
m
\end{array}\right) \frac{(-x)^{m}}{m !}
$$

The diagonalization follows from the following quadratic formula

$$
\sum_{n=0}^{\infty} e^{-\frac{x}{2}} L_{n}(x) e^{-\frac{y}{2}} L_{n}(y) u^{2 n+1}=\frac{u}{1-u^{2}} e^{-\frac{1}{2} \frac{1+u^{2}}{1-u^{2}}(x+y)} I_{0}\left(2 \frac{u \sqrt{x y}}{1-u^{2}}\right),
$$

valid for $u<1$. Hence upon setting $g=1 /(q+1 / q)$, and defining $\alpha=x\left(1+u^{2}\right) /\left(1-u^{2}\right)$, $\alpha^{\prime}=y\left(1+u^{2}\right) /\left(1-u^{2}\right)$, with $u=q$, eqn. (3.16) translates into

$$
G^{(0)}\left(\alpha, \alpha^{\prime}\right)=\sum_{n=0}^{\infty} \psi_{n}^{(0)}(\alpha) \psi_{n}^{(0)}\left(\alpha^{\prime}\right) \lambda_{n}
$$

in which $\psi_{n}^{(0)}(\alpha)$ is the normalized eigenfunction for the eigenvalue $\lambda_{n}$, respectively reading

$$
\begin{aligned}
\psi_{n}^{(0)}(\alpha) & =\sqrt{\frac{1-q^{2}}{1+q^{2}}} e^{-\frac{1}{2} \frac{1-q^{2}}{1+q^{2}} \alpha} L_{n}\left(\frac{1-q^{2}}{1+q^{2}} \alpha\right), \\
\lambda_{n} & =\frac{q^{2 n+1}}{g}, \\
q & =g C\left(g^{2}\right),
\end{aligned}
$$

with $C$ as in (2.6).

This leads immediately to the pure Lorentzian triangulation partition function on a time cylinder with $t$ steps

$$
Z^{(0)}(t)=\sum_{n=0}^{\infty} \lambda_{n}^{t}=\frac{1}{g^{t}} \frac{q^{t}}{1-q^{2 t}}=\frac{C\left(g^{2}\right)^{t}}{1-\left[g C\left(g^{2}\right)\right]^{2 t}} .
$$

Similarly, we get the one-point correlation of the observable $\Sigma_{s}(z)$ as in (3.13) by using the formula (3.16) with $x=y=\alpha\left(1-q^{2}\right) /\left(1+q^{2}\right)$ and $u=q^{t}$ :

$$
\left\langle\Sigma_{s}(z)\right\rangle_{\Theta}=\frac{1}{1+z}\left\langle e^{\frac{\alpha z}{1+z}}\right\rangle_{G^{(0)}}=\frac{I(z)}{I(0)}
$$

where

$$
I(z)=\frac{1}{1+z} \int_{0}^{\infty} d \alpha e^{\alpha\left(\frac{z}{1+z}-\frac{1+q^{2 t}}{1-q^{2 t}} \frac{1-q^{2}}{1+q^{2}}\right)} I_{0}\left(2 \alpha \frac{q^{t}}{1-q^{2 t}} \frac{1-q^{2}}{1+q^{2}}\right) .
$$


Performing explicitly the integral, we find

$$
\left\langle\Sigma_{s}(z)\right\rangle_{\Theta}=\frac{1}{\sqrt{1+z-z \frac{1+q^{t}}{1-q^{t}} \frac{1+q^{2}}{1-q^{2}}}} \frac{1}{\sqrt{1+z-z \frac{1-q^{t}}{1+q^{t}} \frac{1+q^{2}}{1-q^{2}}}} .
$$

The expressions (3.19) and (3.22) have a singularity at $q=1$, i.e. $g=1 / 2$. In order to obtain a continuum theory, we therefore write

$$
2 g=1-\frac{1}{2} a^{2} \Lambda
$$

where $a^{2}$ is a scaling parameter with the dimension of area and where $\Lambda$ is the renormalized fugacity per triangle. Using this scaling for $g$ we have

$$
q=g C\left(g^{2}\right) \sim e^{-a \sqrt{\Lambda}}
$$

and we see that in order to obtain a finite expression for $Z^{(0)}(t)$ in the continuum limit we must set

$$
t=\frac{T}{a}
$$

This gives the following continuum partition function $Z_{T}^{(0)}$

$$
Z_{T}^{(0)} \equiv \lim _{a \rightarrow 0} \frac{1}{2^{t}} Z^{(0)}(t)=\frac{e^{-\sqrt{\Lambda} T}}{1-e^{-2 \sqrt{\Lambda} T}}
$$

Similarly, we must take

$$
z=a \sqrt{\Lambda} \mathcal{Z} \quad \text { and } \quad N=\frac{\mathcal{N}}{a}
$$

where the factor $\sqrt{\Lambda}$ is simply for convenience ( $\mathcal{Z}$ is dimensionless), and substitute this into (3.22) to get the scaled average

$$
\left\langle e^{\sqrt{\Lambda} \mathcal{N} \mathcal{Z}}\right\rangle=\frac{1}{\sqrt{1-2 \mathcal{Z} \operatorname{cotanh}(\sqrt{\Lambda} T)+\mathcal{Z}^{2}}},
$$

generating the moments $\left\langle\mathcal{N}^{k}\right\rangle$ of the (rescaled) number of triangles per time slice. These moments read

$$
\left\langle\mathcal{N}^{k}\right\rangle=\frac{k !}{\Lambda^{\frac{k}{2}}} P_{k}(\operatorname{cotanh}(\sqrt{\Lambda} T))=\frac{k !}{\Lambda^{\frac{k}{2}}} \frac{\sum_{m=0}^{k}\left(\begin{array}{l}
k \\
m
\end{array}\right)^{2} e^{-2 m \sqrt{\Lambda} T}}{\left(1-e^{-2 \sqrt{\Lambda} T}\right)^{k}},
$$

where $P_{k}(x)$ denotes the $k$-th Legendre polynomial. 


\subsection{Example 2: the case $\beta=\infty$}

For $\beta \rightarrow \infty$ the continuum transfer kernel factorizes as follows

$$
G_{\infty, g \theta}\left(\alpha, \alpha^{\prime}\right)=e^{-\frac{\alpha}{2}} I_{0}(2 g \theta \alpha) \times e^{-\frac{\alpha^{\prime}}{2}} I_{0}\left(2 g \theta \alpha^{\prime}\right) .
$$

The partition function therefore factorizes too and reads

$$
\begin{aligned}
& Z^{(\infty)}(t)=\left(Z^{(\infty)}(1)\right)^{t}, \\
& Z^{(\infty)}(1)=\int_{0}^{\infty} d \alpha e^{-\alpha} I_{0}(2 g \theta \alpha)^{2}=\frac{2}{\pi} K\left((4 g \theta)^{2}\right),
\end{aligned}
$$

where $K(x)$ is the complete elliptic integral of the first kind. Note that $Z^{(\infty)}(1)$ is simply the partition function of two (upper and lower) interlocking arch systems connecting points by pairs along a single time-line. The function $K(x)$ is singular at $x=1$. In order to define a continuum theory we would therefore in this case set

$$
4 g \theta=1-a^{2} \Lambda .
$$

Then we get

$$
Z^{(\infty)}(1)=\frac{2}{\pi} K\left(\left(1-a^{2} \Lambda\right)^{2}\right)=-\frac{1}{\pi} \log \left(a^{2} \Lambda\right)+\mathcal{O}(1),
$$

and the leading singular behavior of $Z^{(\infty)}(t)$ is therefore

$$
Z^{(\infty)}(t) \sim\left(-\frac{1}{\pi} \log \left(a^{2} \Lambda\right)\right)^{t} .
$$

Thus, in this case it is not possible to define a sensible continuum time variable.

\section{Scaling regimes}

It is obvious that the possible singularities of $Z(t)$ must come from large Schwinger parameters, $\alpha_{r}$, since the Bessel functions $I_{k}(2 x)$ are polynomial for small $x$ and behave for large $x$ as

$$
I_{k}(2 x) \simeq \frac{e^{2 x}}{\sqrt{4 \pi x}}\left(1-\frac{4 k^{2}-1}{16 x}+O\left(\frac{1}{x^{2}}\right)\right) .
$$

We will therefore be interested in determining the asymptotic behavior of the transfer kernel $G_{\beta, g \theta}\left(\alpha, \alpha^{\prime}\right)$ as $\alpha, \alpha^{\prime} \rightarrow \infty$. It turns out that one has three different scaling regimes corresponding respectively to $\beta<1, \beta=1$ and $\beta>1$. 


\subsection{Three scaling regimes}

When $\beta>1$, the transition function $\phi_{\beta}(x, y)$ in (3.11) is expressed as an absolutely convergent series (3.10), that behaves for large $x$ and $y$ as

$$
\phi_{\beta}(x, y) \sim \frac{e^{2(x+y)}}{4 \pi \sqrt{x y}} \sum_{k \geq 0} \frac{1}{\beta^{2 k}}=\frac{\beta^{2}}{\beta^{2}-1} \frac{e^{2(x+y)}}{4 \pi \sqrt{x y}} .
$$

In order to determine the asymptotic behavior of $\phi_{\beta}(x, y)$ for the remaining values of $\beta$ we make use of the generating function for modified Bessel functions of the first kind

$$
e^{x\left(t+\frac{1}{t}\right)}=I_{0}(2 x)+\sum_{k \geq 1}\left(t^{k}+\frac{1}{t^{k}}\right) I_{k}(2 x) .
$$

From this relation it follows that

$$
\begin{aligned}
\oint \frac{d t}{2 i \pi t} e^{x\left(t+\frac{1}{t}\right)+y\left(\beta^{2} t+\frac{1}{\beta^{2} t}\right)} & =\phi_{\beta}(x, y)+\phi_{\frac{1}{\beta}}(x, y)-I_{0}(2 x) I_{0}(2 y) \\
& =I_{0}\left(2 \sqrt{\left(x+\beta^{2} y\right)\left(x+y / \beta^{2}\right)}\right) .
\end{aligned}
$$

For $\beta=1$ we immediately get from (4.4)

$$
\phi_{1}(x, y)=\frac{1}{2}\left(I_{0}(2(x+y))+I_{0}(2 x) I_{0}(2 y)\right),
$$

which behaves for large $x$ and $y$ as

$$
\phi_{1}(x, y) \sim \frac{e^{2(x+y)}}{4 \sqrt{\pi(x+y)}} .
$$

Finally, when $\beta<1$, we write (4.4) as

$$
\phi_{\beta}(x, y)=I_{0}\left(2 \sqrt{\left(x+\beta^{2} y\right)\left(x+y / \beta^{2}\right)}\right)+I_{0}(2 x) I_{0}(2 y)-\phi_{\frac{1}{\beta}}(x, y),
$$

and notice that since $\phi_{\frac{1}{\beta}}(x, y)$ is now the absolutely convergent series, the last two terms in (4.7) behave like $e^{2(x+y)}$. Using moreover that

$$
\sqrt{\left(x+\beta^{2} y\right)\left(x+y / \beta^{2}\right)}>x+y,
$$

for all real $\beta \neq 1$, the large $x, y$ asymptotics of $\phi_{\beta}(x, y)$ is entirely governed by the first term on the rhs. of (4.7). This means that for $\beta<1$ we have the following asymptotic behavior of the transition function as $x, y \rightarrow \infty$

$$
\phi_{\beta}(x, y) \sim \frac{e^{2 \sqrt{\left(x+\beta^{2} y\right)\left(x+y / \beta^{2}\right)}}}{2 \sqrt{\pi \sqrt{\left(x+\beta^{2} y\right)\left(x+y / \beta^{2}\right)}}}\left(1+\frac{1}{16 \sqrt{\left(x+\beta^{2} y\right)\left(x+y / \beta^{2}\right)}}+\ldots\right) .
$$


To summarize, we have found the following dominant behavior for the transfer kernel $G_{\beta, g \theta}\left(\alpha, \alpha^{\prime}\right) \sim e^{-S_{\beta, g \theta}\left(\alpha, \alpha^{\prime}\right)}$ as $\alpha, \alpha^{\prime} \rightarrow \infty$.

$$
\begin{aligned}
& \beta \geq 1: S_{\beta, g \theta}\left(\alpha, \alpha^{\prime}\right)=\frac{1}{2}\left(\alpha+\alpha^{\prime}\right)(1-4 g \theta), \\
& \beta<1: S_{\beta, g \theta}\left(\alpha, \alpha^{\prime}\right)=\frac{1}{2}\left(\alpha+\alpha^{\prime}\right)-2 g \theta \sqrt{\left(\alpha+\frac{\alpha^{\prime}}{\beta^{2}}\right)\left(\alpha+\beta^{2} \alpha^{\prime}\right)},
\end{aligned}
$$

where the cases $\beta>1$ and $\beta=1$ differ by sub-leading corrections. A crucial difference between $\beta<1$ and $\beta \geq 1$ is that in the latter case the partition function is factorized (at leading order), while in the former case it will be dominated by correlated $\alpha$ 's all of the same order. This in turn reflects the natural property that for low arch densities, successive time slices have lengths of the same order, while the presence of many arches allows for decorrelated lengths.

The following three sections are devoted to the three cases $\beta=1, \beta>1$ and $\beta<1$ for finite $t$. In all cases, we will need the large- $\alpha$ asymptotics of the product of $t$ transfer kernels which may be expressed as $e^{-S_{\beta}} \times U_{\beta}$, with an action $S_{\beta}\left(\left\{\alpha_{s}\right\}\right)$, reading

$$
S_{\beta}\left(\left\{\alpha_{s}\right\}\right)=\sum_{s=1}^{t} S_{\beta, g \theta}\left(\alpha_{s}, \alpha_{s+1}\right),
$$

where $\alpha_{t+1} \equiv \alpha_{1}$, and a non-exponential factor $U_{\beta}\left(\left\{\alpha_{s}\right\}\right)$, which will be detailed below.

\subsection{The case $\beta=1$}

The action $S_{1}\left(\left\{\alpha_{s}\right\}\right)$ and the pre-factor $U_{1}\left(\left\{\alpha_{s}\right\}\right)$ read respectively

$$
S_{1}\left(\left\{\alpha_{s}\right\}\right)=(1-4 g \theta) \sum_{i=1}^{t} \alpha_{s},
$$

and

$$
U_{1}\left(\left\{\alpha_{s}\right\}\right)=\frac{1}{(16 \pi g \theta)^{\frac{t}{2}}} \prod_{s=1}^{t} \frac{1}{\sqrt{\alpha_{s}+\alpha_{s+1}}} .
$$

From the action (4.12), we immediately find the singularity of $Z(t)$ to be at

$$
4 g \theta=1
$$

Furthermore, writing $4 g \theta=1-a^{2} \Lambda$ and performing the change of variables $\alpha_{s}=\beta_{s} / a^{2}$ in the integral (3.9) with $\beta=1$, we get

$$
Z(t) \sim \frac{1}{a^{t}} \int_{0}^{\infty} \prod_{s=1}^{t} \frac{d \beta_{s} e^{-\Lambda \beta_{s}}}{2 \sqrt{\pi} \sqrt{\beta_{s}+\beta_{s+1}}},
$$


when $a \rightarrow 0$. Note that the multiple integral converges and gives a non-trivial dependence on $t$.

This calculation should be compared to that of [5] computing the singularity structure of the $t$-slice "watermelon" correlator of the $O(n)$ model coupled to ordinary 2D Euclidean gravity. Indeed, viewing the slices of watermelon as time-slice separators, the correlator becomes in the limit $n \rightarrow 0$ equivalent to a periodic time Lorentzian partition function with $h=\theta$ and with slightly different boundary conditions (no escaping arches). In both cases the singularity of the partition function is factorized, leading in the case of ref. [5] to a different behavior $Z \sim a^{t}$, in agreement with the KPZ scaling 10.

\subsection{The case $\beta>1$}

In this case, the action is the same as in the $\beta=1$ case $S_{\beta}=S_{1}$ (4.12), while the pre-factor $U_{\beta}\left(\left\{\alpha_{s}\right\}\right)$ reads

$$
U_{\beta}\left(\left\{\alpha_{s}\right\}\right)=\left(\frac{\beta^{2}}{\left(\beta^{2}-1\right) 4 \pi g \theta}\right)^{t} \prod_{s=1}^{t} \frac{1}{\alpha_{s}}
$$

The critical point is therefore the same as in the case $\beta=1$, namely

$$
4 g \theta=1
$$

and setting again $4 g \theta=1-a^{2} \Lambda$, the singularity of $Z(t)$ is factorized with the result

$$
Z(t) \sim\left(-\frac{1}{\pi} \frac{\beta^{2}}{\beta^{2}-1} \log \left(a^{2} \Lambda\right)\right)^{t}
$$

which, up to a proportionality factor, is identical to the behavior at $\beta=\infty$ (3.34).

We may infer that this behavior is characteristic of the $\beta>1$ regime, and breaks down at $\beta=1$ as (4.18) indicates. Once again, for $\beta>1$, it is not possible to introduce a sensible continuum time variable.

\subsection{The case $\beta<1$}

In this last case, the dominant action reads

$$
S_{\beta}\left(\left\{\alpha_{s}\right\}\right)=\sum_{s=1}^{t} \alpha_{s}-2 g \theta \sum_{s=1}^{t} \sqrt{\left(\alpha_{s}+\beta^{2} \alpha_{s+1}\right)\left(\alpha_{s}+\frac{\alpha_{s+1}}{\beta^{2}}\right)},
$$


and the sub-leading factor is

$$
\begin{aligned}
U_{\beta}\left(\left\{\alpha_{s}\right\}\right)= & \frac{1}{(4 \pi g \theta)^{\frac{t}{2}}} \prod_{s=1}^{t} \frac{1}{\left(\left(\alpha_{s}+\beta^{2} \alpha_{s+1}\right)\left(\alpha_{s}+\frac{\alpha_{s+1}}{\beta^{2}}\right)\right)^{\frac{1}{4}}} \times \\
& \left(1+\frac{1}{16 g \theta \sqrt{\left(\alpha_{s}+\beta^{2} \alpha_{s+1}\right)\left(\alpha_{s}+\frac{\alpha_{s+1}}{\beta^{2}}\right)}}+\ldots\right),
\end{aligned}
$$

where in both expressions we assume periodic boundary conditions for the $\alpha$ 's, i.e. $\alpha_{t+1}=$ $\alpha_{1}$. Let us now turn to studying the singular behavior of $Z(t)$. To obtain the critical point, we notice that the dominant action (4.19) is homogeneous of degree one in the $\alpha$ 's, thus in order for the partition function to be defined, all configurations $\{\alpha\}$ must satisfy $S_{\beta}(\{\alpha\}) \geq 0$. The critical point corresponds to the existence of configurations $\left\{\alpha_{*}\right\}$ minimizing the action, while satisfying $S_{\beta}\left(\left\{\alpha_{*}\right\}\right)=0$. These can be obtained from the saddle-point equations

$$
1-\frac{g \theta}{\beta}\left(\beta^{2} x_{s}+\frac{1}{x_{s}}+x_{s-1}+\frac{\beta^{2}}{x_{s-1}}\right)=0, \quad s=1,2, \ldots, t,
$$

where

$$
x_{s}=\left(\frac{\alpha_{s}+\beta^{2} \alpha_{s+1}}{\beta^{2} \alpha_{s}+\alpha_{s+1}}\right)^{1 / 2}, \quad x_{s+t} \equiv x_{s} \text { and } \alpha_{s+t}=\alpha_{s} .
$$

The only periodic solution $\alpha_{s}$ to the above corresponds to $x_{s}=$ const. $=1$ for all $s$, implying that all the $\alpha$ 's must be equal, and moreover this forces $g \theta$ to take the critical value

$$
2 g \theta\left(\beta+\frac{1}{\beta}\right)=1
$$

As expected the action at the saddle-point vanishes automatically.

To capture the singularity of $Z(t)$, let us explore the vicinity of the critical point (4.23). In analogy with the pure triangulation case, we set

$$
2 g \theta\left(\beta+\frac{1}{\beta}\right)=1-\frac{1}{2} a^{2} \Lambda,
$$

where $a^{2}$ is a small parameter with the dimension of area. The homogeneous nature of the saddle-point above, where all the $\alpha$ 's are equal, suggests that we keep, say $\alpha_{1}$, unchanged, and that we perform the change of variables $\alpha_{s}=\alpha_{1}+\xi_{s} \sqrt{\alpha_{1}}$ for $s=2,3, \ldots, t$. Then the sub-leading factor (4.20) together with the integration measure behave for large $\alpha_{1}$ as

$$
\prod_{s=1}^{t} d \alpha_{s} U_{\beta}\left(\left\{\alpha_{s}\right\}\right) \simeq \frac{d \alpha_{1}}{\sqrt{2 \pi \alpha_{1}}} \prod_{s=2}^{t} \frac{d \xi_{s}}{\sqrt{2 \pi}},
$$


while the action reads

$$
\begin{aligned}
S\left(\alpha_{1},\{\xi\}\right) & =t \alpha_{1}+\sqrt{\alpha_{1}} \sum_{s=1}^{t} \xi_{s} \\
& -\left(1-\frac{1}{2} a^{2} \Lambda\right) \alpha_{1} \sum_{s=1}^{t} \sqrt{\left(1+\frac{1}{\sqrt{\alpha_{1}}} \frac{\xi_{s}+\beta^{2} \xi_{s+1}}{1+\beta^{2}}\right)\left(1+\frac{1}{\sqrt{\alpha_{1}}} \frac{\xi_{s+1}+\beta^{2} \xi_{s}}{1+\beta^{2}}\right)} \\
& =\frac{a^{2}}{2} \Lambda t \alpha_{1}+\frac{1}{8}\left(\frac{1-\beta^{2}}{1+\beta^{2}}\right)^{2} \sum_{s=1}^{t}\left(\xi_{s+1}-\xi_{s}\right)^{2}+O\left(\frac{1}{\sqrt{\alpha_{1}}}\right)
\end{aligned}
$$

where we have set $\xi_{1}=\xi_{t+1}=0$. To evaluate $Z(t)$, we must first integrate over the $\xi$ 's (along the real line) and then over $\alpha_{1}>0$. This results in

$$
\begin{aligned}
Z(t) & \sim \int_{0}^{\infty} \frac{d \alpha_{1}}{\sqrt{2 \pi \alpha_{1}}} e^{-\frac{1}{2} a^{2} \Lambda t \alpha_{1}}\left(2 \frac{1+\beta^{2}}{1-\beta^{2}}\right)^{t-1} t^{-1 / 2} \\
& =\frac{1-\beta^{2}}{2 a \sqrt{\Lambda} t\left(1+\beta^{2}\right)}\left(2 \frac{1+\beta^{2}}{1-\beta^{2}}\right)^{t}
\end{aligned}
$$

where the $\xi$ integral has produced the term $t^{-1 / 2}=\operatorname{det}(\Delta)^{-1 / 2}$, where $\Delta$ is the $(t-1) \times$ $(t-1)$ matrix of the discrete Laplacian, namely $\Delta_{r, s}=2 \delta_{r, s}-\delta_{r, s+1}-\delta_{r, s-1}$, truncated to $1 \leq r, s \leq t-1$. Note that in (4.27) we have displayed the trivial entropic factor $\left(2\left(1+\beta^{2}\right) /\left(1-\beta^{2}\right)\right)^{t}$, namely a weight 2 for each application of $\Theta^{(1)}$ and $\left(1+\beta^{2}\right) /\left(1-\beta^{2}\right)$

for each application of $\Theta^{(2)}$. These factors could of course be absorbed in a redefinition $\Theta^{(1)} \rightarrow \Theta^{(1)} / 2$ and $\Theta^{(2)} \rightarrow \Theta^{(2)} /\left(\frac{1+\beta^{2}}{1-\beta^{2}}\right)$.

\section{The scaling limit and the Calogero Hamiltonian}

In order to obtain a finite result when $a \rightarrow 0$ for the partition function (4.27) (after stripping it from the trivial entropic factor), we must let simultaneously $t$ scale as $1 / a$. We therefore introduce a continuum time variable $T$ through

$$
t=\frac{T}{a}
$$

We note that (5.1) is the same scaling of the time variable as in the pure case (cf. eqn. (3.25)). This means in particular that the fractal dimension, $d_{H}$ of our surfaces for $\beta<1$ is also the same as in the pure Lorentzian case, i.e. $d_{H}=2$. 


\subsection{Partition function}

Let us now compute the partition function of the $\beta<1$ model in the vicinity of the critical point using (4.24) and (5.1) and letting $a \rightarrow 0$. We know already that the integral (3.9) should be dominated in this limit by large $\alpha$ 's that are close to one another. This suggests that we directly perform the following change of variables in the integral

$$
\alpha_{s}=\frac{\varphi_{s}^{2}}{a}, \quad \varphi_{s}>0
$$

Inserting the change of variables in the action (4.19) leads to

$$
\begin{aligned}
S(\varphi) & =\frac{1}{a} \sum_{s=1}^{t} \varphi_{s}^{2}-\frac{\left(1-\frac{1}{2} a^{2} \Lambda\right)}{a} \sum_{s=1}^{t} \varphi_{s}^{2} \sqrt{\left(1+\frac{\varphi_{s+1}^{2}-\varphi_{s}^{2}}{\varphi_{s}^{2}\left(1+\beta^{2}\right)}\right)\left(1+\beta^{2} \frac{\varphi_{s+1}^{2}-\varphi_{s}^{2}}{\varphi_{s}^{2}\left(1+\beta^{2}\right)}\right.} \\
& \simeq \frac{1}{2} a \Lambda \sum_{s=1}^{t} \varphi_{s}^{2}+\frac{1}{8 a}\left(\frac{1-\beta^{2}}{1+\beta^{2}}\right)^{2} \sum_{s=1}^{t} \frac{1}{\varphi_{s}^{2}}\left(\varphi_{s+1}^{2}-\varphi_{s}^{2}\right)^{2},
\end{aligned}
$$

where both terms are of order $a$, as $\varphi_{s+1}^{2}-\varphi_{s}^{2}$ is of order $a$. The contribution from the pre-factor $U_{\beta}\left(\left\{\alpha_{s}\right\}\right)$ (4.20) and the measure reads

$$
\prod_{s=1}^{t} d \alpha_{s} U_{\beta}\left(\left\{\alpha_{s}\right\}\right) \simeq \prod_{s=1}^{t} \sqrt{\frac{2}{\pi a}} d \varphi_{s}\left(1+\frac{a}{8 \varphi_{s}^{2}}\right) \simeq\left(\prod_{s=1}^{t} \frac{2}{\sqrt{2 \pi a}} d \varphi_{s}\right) e^{\frac{a}{8} \sum_{s=1}^{t} \frac{1}{\varphi_{s}^{2}}}
$$

We notice that this latter relation has the effect of adding an extra term to the action (5.3). Next, we introduce a discrete function by $\varphi(u=s / t) \equiv \varphi_{s}$ and we assume that for large $t$ (small $a$ ) this function becomes a smooth function of the continuous variable $u \in[0,1]$. This allows us to Taylor-expand $\varphi_{s+1}$ around $\varphi_{s}$ as $\varphi(u+a / T)=\varphi(u)+$ $\frac{a}{T} \varphi^{\prime}(u)+\mathcal{O}\left(a^{2}\right)$. Furthermore, we can replace sums by integrals, $\sum_{s} \rightarrow \frac{T}{a} \int_{0}^{1} d u$. Finally, it proves convenient to define the continuum partition function $Z_{T}$ by

$$
Z_{T} \equiv \lim _{a \rightarrow 0}\left(\frac{1}{2} \frac{1-\beta^{2}}{1+\beta^{2}}\right)^{t} Z(t)
$$

Inserting everything and rescaling $\varphi \rightarrow\left(\frac{1+\beta^{2}}{1-\beta^{2}}\right) \varphi, u \rightarrow T u$ we get

$$
Z_{T}=\int_{\varphi(0)=\varphi(T)} \mathcal{D} \varphi e^{-\frac{1}{2} \int_{0}^{T} d u\left(\varphi^{\prime}(u)^{2}+\omega^{2} \varphi(u)^{2}-\frac{A}{4 \varphi(u)^{2}}\right)}
$$

where we have identified the functional measure as

$$
\mathcal{D} \varphi=\lim _{a \rightarrow 0, t=\frac{T}{a} \rightarrow \infty} \prod_{s=1}^{t} \frac{d \varphi_{s}}{\sqrt{2 \pi a}}
$$


and where the integration extends over real positive fields $\varphi(u)$ defined for $u \in[0, T]$ and obeying $\varphi(0)=\varphi(T)$. The parameters $\omega$ and $A$ are given by

$$
\omega=\sqrt{\Lambda}\left(\frac{1+\beta^{2}}{1-\beta^{2}}\right), \quad A=\left(\frac{1-\beta^{2}}{1+\beta^{2}}\right)^{2} .
$$

Using the Feynman-Kac formula we see that computing $Z_{T}$ amounts to solving a onedimensional (imaginary time) quantum mechanical system on the real half-line, namely

$$
Z_{T}=\operatorname{Tr}\left(e^{-T \hat{H}}\right)
$$

with a Hamiltonian

$$
\hat{H}=-\frac{1}{2} \frac{d^{2}}{d \varphi^{2}}+\frac{1}{2} \omega^{2} \varphi^{2}-\frac{A}{8 \varphi^{2}}, \quad \varphi>0
$$

This is nothing but the celebrated Calogero Hamiltonian for one particle. We thus arrive at the result that $1+1$-dimensional Lorentzian triangulations (in the low arch density regime $\beta<1$ ) are equivalent in the continuum limit to a one-dimensional Calogero model. This holds in particular for the pure case. A Hamiltonian describing the continuum limit of pure Lorentzian triangulations, i.e. $\beta \rightarrow 0$ was derived in ref. [3] using a strategy different from the one employed here. The Hamiltonian of ref. [3] is expressed in terms of a variable $L$ which has the interpretation of the continuum counterpart of the length $i$ of a line of constant time. As we shall see later the field $\varphi^{2}$ has the interpretation of the continuum counterpart of the total number of triangles in a given time slice $i+j$, also equal to the total length of the two adjacent constant time lines, translating into $2 L$ in the continuum limit. Due to the use of different boundary conditions in ref. [3] and here one cannot immediately compare the two Hamiltonians. In appendix A we show how to recover the Calogero Hamiltonian for $\beta \rightarrow 0$ using the approach of ref. [3]. As expected a change of variables $2 L \rightarrow \varphi^{2}$ is involved. We also explain why the approach of ref. [3] does not extend to $\beta \neq 0$.

Note that the parameter $A$ of our Hamiltonian (5.10) satisfies $0 \leq A \leq 1$ and that $A=$ 1 iff $\beta=0$. It is well-known [11] that $A=1$ is a limiting case for the quantum mechanical system (5.10). For $A>1$ the operator $\hat{H}$ is no longer self-adjoint. Our generalized Lorentzian triangulation model thus always leads to a physically acceptable Hamiltonian. For $\beta<1$ the Hamiltonian $\hat{H}$ is readily diagonalized as follows [11]. Introducing the confluent hypergeometric function

$$
F(a, b, x)=\sum_{k=0}^{\infty} \frac{a(a+1) \ldots(a+k-1)}{k ! b(b+1) \ldots(b+k-1)} x^{k},
$$


the eigenvectors of $\hat{H}$ take the form

$$
\psi_{n}(\varphi)=\mathcal{A}_{n} e^{-\frac{1}{2} \omega \varphi^{2}} \varphi^{\mu-\frac{1}{2}} F\left(-n, \mu, \omega \varphi^{2}\right)
$$

where $\mathcal{A}_{n}$ is a normalization factor

$$
\mathcal{A}_{n}=\sqrt{\frac{2 \omega^{\mu} \Gamma(n+\mu)}{\Gamma(n+1) \Gamma(\mu)^{2}}},
$$

ensuring the orthonormality of the eigenbasis $\psi_{n}(\varphi)$, and the parameter $\mu$ may take two values, related to the potential strength $A$ through $\mu=1 \pm \frac{1}{2} \sqrt{1-A}$. However, the smaller of these two values must be discarded as unphysical [11], leading to

$$
\mu=1+\frac{\beta}{1+\beta^{2}} .
$$

Note that the confluent hypergeometric series $F$ is truncated by the value $a=-n$ to a polynomial of degree $n$, generalizing the Laguerre polynomial $L_{n}$, namely

$$
F(-n, \mu, z)=\sum_{k=0}^{n}(-z)^{k}\left(\begin{array}{l}
n \\
k
\end{array}\right) \frac{1}{\mu(\mu+1) \ldots(\mu+k-1)} .
$$

The corresponding eigenvalues read

$$
E_{n}=\omega(2 n+\mu)=\omega\left(2 n+1+\frac{\beta}{1+\beta^{2}}\right), \quad n=0,1,2, \ldots
$$

This leads to the scaled partition function

$$
Z_{T}=\sum_{n \geq 0} e^{-T E_{n}}=\frac{e^{-\sqrt{\Lambda} T\left(\frac{1+\beta+\beta^{2}}{1-\beta^{2}}\right)}}{1-e^{-2 \sqrt{\Lambda} T\left(\frac{1+\beta^{2}}{1-\beta^{2}}\right)}} .
$$

For $\beta \rightarrow 0$ the result (5.17) reduces to the pure Lorentzian triangulation result (3.26). On the level of eigenvalues and eigenfunctions we have $\lim _{a \rightarrow 0}\left(g \lambda_{n}\right)^{t}=\lim _{\mu \rightarrow 1} e^{-E_{n} T}$ and $\lim _{a \rightarrow 0} \psi_{n}^{(0)}(\alpha) \sqrt{d \alpha}=\lim _{\mu \rightarrow 1} \psi_{n}(\varphi) \sqrt{d \varphi}$, respectively obtained from eqns. (3.18) and (5.12) with $\omega \rightarrow \sqrt{\Lambda}$, and after setting $q=e^{-a \sqrt{\Lambda}}, t=\frac{T}{a}$ and $\alpha=\frac{\varphi^{2}}{a}$ as before.

Furthermore, we see that as $\beta \rightarrow 1$ the exponents in (5.17) become singular, signaling another type of scaling coming into play (c.f. Sect. 4.2 and 4.3 above). It is worth stressing that the parameter $\beta$ cannot be absorbed by a redefinition of the renormalized triangle fugacity $\Lambda$ or the continuum time variable, and is itself a genuine continuum parameter. This will prove even more visible when we compute correlation functions. Such a situation where a coupling constant introduced at the discrete level survives in an unrenormalized form in the continuum limit is quite unusual. 


\subsection{The loop-loop correlator}

The Laplace transform of the loop-loop correlator $Z(x, y, t)$ can be written as (cf. eqn. (2.8)

$$
\begin{aligned}
Z(x, y, t)= & \sum_{i, j \geq 0} Z(i, j, t) x^{i} y^{j} \\
= & \prod_{s=1}^{t}\left(\oint_{\mathcal{C}} \frac{d w_{s}}{2 i \pi w_{s}} \frac{d z_{s}}{2 i \pi z_{s}}\right) \times \\
& \Theta^{(1)}\left(x, \frac{1}{w_{1}}\right)\left(\prod_{r=1}^{t-1} \Theta^{(2)}\left(w_{r}, z_{r}\right) \Theta^{(1)}\left(\frac{1}{z_{r}}, \frac{1}{w_{r+1}}\right)\right) \Theta^{(2)}\left(w_{t}, z_{t}\right) \Theta^{(1)}\left(\frac{1}{z_{t}}, y\right) .
\end{aligned}
$$

Repeating the computation of Sect. 3, namely performing the change of variables $w_{r}, z_{r} \rightarrow$ $\mu_{r}, \rho_{r}$ (cf. eqns. (3.2) and (3.4)), representing the $\Theta^{(1)}$-factors as Schwinger-type integrals, and performing the explicit contour integrals over the $\rho_{r}$ and $\mu_{r}$, it appears that $x$ and $y$ are simply spectators throughout the computation, and one finds

$$
Z(x, y, t)=\prod_{s=0}^{t}\left(\int_{0}^{\infty} d \alpha_{s} e^{-\alpha_{s}}\right) e^{g\left(\alpha_{0} x+\alpha_{t} y\right)} \prod_{r=0}^{t-1} \phi_{\beta}\left(g \theta \alpha_{r}, g \theta \alpha_{r+1}\right)
$$

where the transition function $\phi_{\beta}(x, y)$ has been defined in (3.10). Apart from the explicit exponential dependence on $x$ and $y$, the loop-loop correlator differs from the partition function (3.9) by involving one more Schwinger parameter. Note also that in this case we do not impose periodic boundary conditions on the $\alpha$ 's.

We will now study the behavior of the loop-loop correlator in the scaling limit defined by (4.24) and (5.1). Here we must also assume that $x$ and $y$ are close to their critical values $x_{c}=y_{c}=\frac{1}{2 g}$ (corresponding to the poles of the $\Theta^{(1)}$-factors in (5.18)). It turns out that the correct scaling ansatz reads

$$
x=\frac{1}{2 g}(1-a X), \quad y=\frac{1}{2 g}(1-a Y) .
$$

To calculate $Z(x, y, t)$ in the scaling limit we use the same strategy as in Sect. 5.1. We perform the change of variables $\alpha_{s}=\frac{\varphi_{s}^{2}}{a}, s=0,1, \ldots, t$, introduce a discrete function by $\varphi(u=s / t) \equiv \varphi_{s}$ and assume that as $t \rightarrow \infty$ this function becomes a smooth function of a continuous variable $u \in[0,1]$. However, in the present case we must deal with fixed as opposed to periodic boundary conditions for $\varphi$ and special care has to be taken in the treatment of the boundary terms. Writing the integrand in (5.19) for large $\alpha_{s}$ as $e^{-S_{\beta}} U_{\beta}$ 
in the same way as in Sect. 3, we get, when inserting our change of variables, the following contribution from the measure and the pre-factor

$$
\left(\prod_{s=0}^{t} d \alpha_{s}\right) U_{\beta}\left(\left\{\alpha_{s}\right\}\right)=\frac{2^{t+1}}{a} \frac{1}{\sqrt{2 \pi a}} d \varphi_{0} \sqrt{\varphi_{0}} d \varphi_{t} \sqrt{\varphi_{t}}\left(\prod_{s=1}^{t-1} \frac{d \varphi_{s}}{\sqrt{2 \pi a}}\right) e^{\frac{a}{8}\left(\frac{1}{2}\left(\frac{1}{\varphi_{0}^{2}}+\frac{1}{\varphi_{t}^{2}}\right)+\sum_{r=1}^{t-1} \frac{1}{\varphi_{r}^{2}}\right)+\mathcal{O}\left(a^{2}\right)}
$$

Inserting the various scaling relations in $S_{\beta}\left(\left\{\alpha_{s}\right\}\right)$ on sees that the scaling ansatz (5.20) is exactly what is needed to make divergent boundary terms cancel. Furthermore it becomes natural to define a continuum loop-loop correlator $Z_{T}(X, Y)$ by the following recipe

$$
Z_{T}(X, Y)=\lim _{a \rightarrow 0} a \cdot \frac{1}{2^{t+1}}\left(\frac{1-\beta^{2}}{1+\beta^{2}}\right)^{t} Z(x, y, t) .
$$

Here we choose to scale away the same entropic factor as in the case of partition function, namely a factor 2 for each occurrence of the transfer matrix $\Theta^{(1)}$ and a factor $\left(\frac{1+\beta^{2}}{1-\beta^{2}}\right)$ for each occurrence of the transfer matrix $\Theta^{(2)}$. Collecting all the terms and rescaling $\varphi \rightarrow\left(\frac{1-\beta^{2}}{1+\beta^{2}}\right) \varphi$ and $u \rightarrow T u$ as before, we find that the continuum loop-loop correlator can be expressed as the quantum mechanical propagator

$$
Z_{T}(X, Y)=\left(\frac{1+\beta^{2}}{1-\beta^{2}}\right)^{2} \int_{0}^{\infty} \sqrt{\varphi_{0}} d \varphi_{0} \sqrt{\varphi_{T}} d \varphi_{T} e^{-\frac{1}{2}\left(\varphi_{0}^{2} \tilde{X}+\varphi_{T}^{2} \tilde{Y}\right)}\left\langle\varphi_{T}\left|e^{-T \hat{H}}\right| \varphi_{0}\right\rangle
$$

where the Hamiltonian $\hat{H}$ is defined as in (5.10), with $\omega$ and $A$ as in (5.8), and where we have set

$$
\tilde{X}=\left(\frac{1+\beta^{2}}{1-\beta^{2}}\right)^{2} X, \quad \tilde{Y}=\left(\frac{1+\beta^{2}}{1-\beta^{2}}\right)^{2} Y .
$$

To evaluate $Z_{T}(X, Y)$ we simply have to insert two decompositions of the identity as a sum over projectors on the eigenspaces of $\hat{H}$, i.e. $I=\sum_{n \geq 0}|n\rangle\langle n|$ and use $\langle n \mid \varphi\rangle=\psi_{n}(\varphi)$, where $\psi_{n}(\varphi)$ is the $n$-th normalized eigenfunction of $\hat{H}$, defined in (5.12). This yields

$$
\begin{aligned}
Z_{T}(X, Y) & =\left(\frac{1+\beta^{2}}{1-\beta^{2}}\right)^{2} \sum_{n=0}^{\infty} e^{-T E_{n}} G_{n}(\tilde{X}) G_{n}(\tilde{Y}), \\
G_{n}(Z) & =\int_{0}^{\infty} \sqrt{\varphi} d \varphi e^{-\frac{1}{2} \varphi^{2} Z} \psi_{n}(\varphi)
\end{aligned}
$$

with $E_{n}$ as in (5.16). The function $G_{n}(Z)$ is readily determined using the expression (5.12) for $\psi_{n}(\varphi)$

$$
G_{n}(Z)=\mathcal{A}_{n} \int_{0}^{\infty} d \varphi \varphi^{\mu} e^{-\frac{1}{2} \varphi^{2}(\omega+Z)} F\left(-n, \mu, \omega \varphi^{2}\right)
$$


where $\mu=1+\frac{\beta}{1+\beta^{2}}$ as before. Inserting the polynomial expression for the truncated confluent hypergeometric function (5.15), eqn. (5.26) can be integrated term by term with the result

$$
\begin{aligned}
G_{n}(Z) & =\frac{\mathcal{A}_{n}}{2}\left(\frac{2}{\omega+Z}\right)^{\frac{\mu+1}{2}} \sum_{k=0}^{n}\left(\begin{array}{l}
n \\
k
\end{array}\right) \frac{\Gamma\left(\frac{\mu+1}{2}+k\right)}{\mu(\mu+1) \ldots(\mu+k-1)}\left(-\frac{2 \omega}{\omega+Z}\right)^{k} \\
& =\frac{\mathcal{A}_{n}}{2} \Gamma\left(\frac{\mu+1}{2}\right)\left(\frac{2}{\omega+Z}\right)^{\frac{\mu+1}{2}} F\left(-n, \frac{\mu+1}{2}, \mu, \frac{2 \omega}{\omega+Z}\right) .
\end{aligned}
$$

Here we have recognized the ordinary hypergeometric function

$$
F(a, b, c, z) \equiv{ }_{2} F_{1}(a, b, c, z)=\sum_{k=0}^{\infty} z^{k} \frac{a(a+1) \ldots(a+k-1) b(b+1) \ldots(b+k-1)}{k ! c(c+1) \ldots(c+k-1)}
$$

Thus, the continuum loop-loop correlator reads

$$
\begin{aligned}
& Z_{T}(X, Y)=\left(\frac{1+\beta^{2}}{1-\beta^{2}}\right)^{2} \frac{1}{2 \omega}\left(\frac{\Gamma\left(\frac{\mu+1}{2}\right)}{\Gamma(\mu)}\right)^{2}\left(\frac{4 \omega^{2}}{(\omega+\tilde{X})(\omega+\tilde{Y})}\right)^{\frac{\mu+1}{2}} \\
& \quad \times \sum_{n=0}^{\infty} e^{-\omega T(2 n+\mu)} \frac{\Gamma(n+\mu)}{\Gamma(n+1)} F\left(-n, \frac{\mu+1}{2}, \mu, \frac{2 \omega}{\omega+\tilde{X}}\right) F\left(-n, \frac{\mu+1}{2}, \mu, \frac{2 \omega}{\omega+\tilde{Y}}\right) .
\end{aligned}
$$

This expression may further be simplified by use of the following quadratic relation due to Meixner [12]

$$
\begin{aligned}
& \sum_{n=0}^{\infty} \frac{\Gamma(\mu+n)}{\Gamma(\mu) n !}(-s)^{n} F(-n, a, \mu, z) F(-n, b, \mu, w) \\
& \quad=\frac{(1+s)^{a+b-\mu}}{(1+s(1-z))^{a}(1+s(1-w))^{b}} F\left(a, b, \mu,-\frac{s z w}{(1+s(1-z))(1+s(1-w))}\right) .
\end{aligned}
$$

Indeed, applying (5.30) with $s=-e^{-2 T \omega}, a=b=\frac{\mu+1}{2}, z=2 \omega /(\omega+\tilde{X})$, and $w=$ $2 \omega /(\omega+\tilde{Y})$, the continuum loop-loop correlator finally reads

$$
Z_{T}(X, Y)=\left(\frac{1+\beta^{2}}{1-\beta^{2}}\right)^{2} \frac{\sinh (\omega T)}{\omega} \frac{\Gamma\left(\frac{\mu+1}{2}\right)^{2}}{\Gamma(\mu)} v^{\frac{\mu+1}{2}} F\left(\frac{\mu+1}{2}, \frac{\mu+1}{2}, \mu, v\right)
$$

where

$$
v=\frac{\omega}{(\tilde{X} \sinh (\omega T)+\omega \cosh (\omega T))} \times \frac{\omega}{(\tilde{Y} \sinh (\omega T)+\omega \cosh (\omega T))}
$$


Let us consider the pure Lorentzian triangulation limit $\beta \rightarrow 0$, i.e. $\mu \rightarrow 1$ and $\omega \rightarrow$ $\sqrt{\Lambda}$. This limit involves the hypergeometric function $F(1,1,1, v)=1 /(1-v)$, and the corresponding loop-loop propagator reads

$$
\begin{aligned}
Z_{T}^{(0)}(X, Y) & =\left.\frac{\sinh (\omega T)}{\omega} \frac{v}{1-v}\right|_{\omega=\sqrt{\Lambda}} \\
& =\frac{\sqrt{\Lambda}}{(X Y+\Lambda) \sinh (\sqrt{\Lambda} T)+\sqrt{\Lambda}(X+Y) \cosh (\sqrt{\Lambda} T)}
\end{aligned}
$$

This coincides precisely with eqn. (2.29) of ref. [9].

Let us now translate the result (5.31) into the language of conjugate rescaled loop lengths by setting

$$
L_{0}=a i, \quad L_{T}=a j, \quad T=a t
$$

in terms of which the rescaled loop-loop correlator reads

$$
Z_{T}\left(L_{0}, L_{T}\right)=\lim _{a \rightarrow 0} \frac{1}{a} \frac{1}{2^{t+1}}\left(\frac{1-\beta^{2}}{1+\beta^{2}}\right)^{t} Z(i, j, t)
$$

This is obtained by taking the inverse Laplace transform of (5.31) over $X$ and $Y$. In practice, it is more convenient to read it directly off the original expression (5.23) upon performing the change of variables

$$
L_{0}=\frac{1}{2} \varphi_{0}^{2}\left(\frac{1+\beta^{2}}{1-\beta^{2}}\right)^{2}, \quad L_{T}=\frac{1}{2} \varphi_{T}^{2}\left(\frac{1+\beta^{2}}{1-\beta^{2}}\right)^{2}
$$

relating $\varphi_{0}^{2}$ resp. $\varphi_{T}^{2}$ to the numbers of triangles pointing up resp. down in the time slice $u=0$ resp. $u=T$. This yields

$$
Z_{T}\left(L_{0}, L_{T}\right)=\left.\left(\frac{1-\beta^{2}}{1+\beta^{2}}\right)^{2} \frac{\left\langle\varphi_{T}\left|e^{-T \hat{H}}\right| \varphi_{0}\right\rangle}{\sqrt{\varphi_{0} \varphi_{T}}}\right|_{\varphi_{0}=\frac{1-\beta^{2}}{1+\beta^{2}} \sqrt{2 L_{0}}, \varphi_{T}=\frac{1-\beta^{2}}{1+\beta^{2}} \sqrt{2 L_{T}}}
$$

To evaluate the above heat kernel, we can use the following quadratic relation satisfied by the generalized Laguerre polynomials introduced in Sect. 5.1, and generalizing the pure case (3.16), namely 13

$$
\sum_{n \geq 0} \frac{\Gamma(n+\mu)}{n ! \Gamma(\mu)^{2}} F(-n, \mu, x) F(-n, \mu, y) z^{n}=\frac{1}{1-z} e^{-\frac{z(x+y)}{1-z}}(x y z)^{\frac{1-\mu}{2}} I_{\mu-1}\left(2 \frac{\sqrt{x y z}}{1-z}\right)
$$


where $I_{\mu-1}(2 x)=\sum_{p \geq 0} x^{\mu-1+2 p} /(p ! \Gamma(p+\mu))$ is the modified Bessel function. This equation is immediately rephrased in terms of the eigenfunctions $\psi_{n}$ of $\hat{H}$ (5.12) as

$$
\sum_{n \geq 0} z^{n+\frac{\mu}{2}} \psi_{n}\left(\varphi_{0}\right) \psi_{n}\left(\varphi_{T}\right)=2 \omega \sqrt{\varphi_{0} \varphi_{T}} \frac{\sqrt{z}}{1-z} e^{-\frac{1+z}{1-z} \frac{\omega}{2}\left(\varphi_{0}^{2}+\varphi_{T}^{2}\right)} I_{\mu-1}\left(2 \frac{\sqrt{z} \omega \varphi_{0} \varphi_{T}}{1-z}\right)
$$

Taking $z=e^{-2 \omega T}$ we deduce the expression for the heat kernel of the Calogero Hamiltonian $\hat{H}$, leading finally to

$$
\begin{aligned}
Z_{T}\left(L_{0}, L_{T}\right)=\left(\frac{1-\beta^{2}}{1+\beta^{2}}\right)^{2} & \frac{\omega}{\sinh (\omega T)} e^{-\left(L_{0}+L_{T}\right)\left(\frac{1-\beta^{2}}{1+\beta^{2}}\right)^{2} \omega \operatorname{cotanh}(\omega T)} \times \\
& \times I_{\mu-1}\left(2\left(\frac{1-\beta^{2}}{1+\beta^{2}}\right)^{2} \frac{\omega}{\sinh (\omega T)} \sqrt{L_{0} L_{T}}\right),
\end{aligned}
$$

with $\omega$ as in (5.8) and $\mu-1=\beta /\left(1+\beta^{2}\right)$. This result coincides exactly with eqn. (2.32) of [9].

\subsection{Correlation functions on a time cylinder}

In this section, we will compute for $\beta<1$ the general correlations (3.13) of the observables $\Sigma_{s_{m}}\left(z_{m}\right)$ (3.12), in the continuum limit where $a \rightarrow 0$, with

$$
s_{m}=\frac{u_{m}}{a} \quad, \quad z_{m}=a \omega \mathcal{Z}_{m} \quad \text { and } \quad \alpha_{s_{m}}=\frac{\varphi^{2}\left(u_{m}\right)}{a} .
$$

With these substitutions, eqn. (3.13) becomes

$$
\left\langle\prod_{m=1}^{k} e^{\omega \mathcal{N}\left(u_{m}\right) \mathcal{Z}_{m}}\right\rangle=\left\langle\prod_{m=1}^{k} e^{\omega \varphi^{2}\left(u_{m}\right) \mathcal{Z}_{m}}\right\rangle
$$

where the rescaled number of triangles in the time-slice $u \in[0, T]$ reads $\mathcal{N}(u)=$ $a\left(\frac{1-\beta^{2}}{1+\beta^{2}}\right)^{2} N(u / a)$. Apart from its instrumentality, eqn. (5.42) yields the interpretation of the field $\varphi(u)$ : the square of $\varphi(u)$ is identified with the rescaled number $\mathcal{N}(u)$. This is very reminiscent of the field theoretical representation of polymers, where $\varphi^{2}$ is identified with the polymer density. For the pure case, this should not come as a surprise as we have shown in [9] that Lorentzian triangulations can be mapped onto random walks.

Let us first compute the one-point average of $e^{\omega \mathcal{N Z}}$ :

$$
f_{\mu}(\mathcal{Z}) \equiv\left\langle e^{\omega \mathcal{N} \mathcal{Z}}\right\rangle=\frac{1}{Z_{T, \mu}} \int_{0}^{\infty} d \varphi \sum_{n \geq 0} \psi_{n}(\varphi)^{2} e^{-\omega T(2 n+\mu)} e^{\mathcal{Z} \omega \varphi^{2}}
$$


with the cylinder partition function $Z_{T, \mu}=e^{-\omega T \mu} /\left(1-e^{-2 \omega T}\right)$, indexed by $\mu=1+\beta /(1+$ $\left.\beta^{2}\right)$ for convenience. We use again the above quadratic equation (5.39) with $z=e^{-2 \omega T}$ and $\varphi_{0}=\varphi_{T}=\varphi$, and we perform the change of variables $u \equiv \omega \varphi^{2}$ to get

$$
f_{\mu}(\mathcal{Z})=e^{\omega T(\mu-1)} \int_{0}^{\infty} d u e^{-(\operatorname{cotanh}(\omega T)-\mathcal{Z}) u} I_{\mu-1}\left(\frac{u}{\sinh (\omega T)}\right) .
$$

This last integral is a particular case of the generic Laplace transform of the modified Bessel [13]:

$$
r \int_{0}^{\infty} d u e^{-r u} I_{\mu-1}(s u)=\frac{(r / s)^{\mu-1}}{\sqrt{1-(s / r)^{2}}}\left(1-\sqrt{1-(s / r)^{2}}\right)^{\mu-1},
$$

and we finally get

$$
f_{\mu}(\mathcal{Z})=\frac{\left(\frac{\operatorname{cotanh}(\omega T)-\mathcal{Z}-\sqrt{1-2 \operatorname{cotanh}(\omega T) \mathcal{Z}+\mathcal{Z}^{2}}}{\operatorname{cotanh}(\omega T)-1}\right)^{\mu-1}}{\sqrt{1-2 \operatorname{cotanh}(\omega T) \mathcal{Z}+\mathcal{Z}^{2}}}
$$

generalizing the pure case result (3.28) corresponding to $\mu=1$ and $\omega=\sqrt{\Lambda}$. This yields alternatively the moments $\left\langle\mathcal{N}^{k}\right\rangle$ of the rescaled number of triangles per time-slice

$$
\left\langle\mathcal{N}^{k}\right\rangle=\frac{k !}{\omega^{k}} P_{k}^{(\mu-1,1-\mu)}(\operatorname{cotanh}(\omega T))=\frac{k !}{\omega^{k}} \frac{\sum_{m=0}^{k}\left(\begin{array}{c}
k+\mu-1 \\
m
\end{array}\right)\left(\begin{array}{c}
k+1-\mu \\
k-m
\end{array}\right) e^{-2 m \omega T}}{\left(1-e^{-2 \omega T}\right)^{k}},
$$

where $P_{k}^{(\alpha, \beta)}(x)$ denotes the $k$-th Jacobi polynomial [13].

The formula (5.46) generalizes nicely to the case of the $k$-point function (5.42), and we leave the details of its derivation to appendix B below. Defining the time intervals

$$
t_{m}=u_{m+1}-u_{m},
$$

for $m=1,2, \ldots, k-1$ and $t_{k}=T-\sum_{1 \leq m \leq k-1} t_{m}$, the result reads

$$
\left\langle\prod_{m=1}^{k} e^{\omega \mathcal{N}\left(u_{m}\right) \mathcal{Z}_{m}}\right\rangle=g_{\mu}\left(\frac{2}{P\left(\mathcal{Z}_{1}, \ldots, \mathcal{Z}_{k} \mid t_{1}, \ldots, t_{k}\right)}\right),
$$

where

$$
g_{\mu}(s)=\sinh (\omega T) e^{\omega T(\mu-1)} s^{2-\mu} \frac{\left(1-\sqrt{1-s^{2}}\right)^{\mu-1}}{\sqrt{1-s^{2}}}=f_{\mu}\left(\operatorname{cotanh}(\omega T)-\frac{1}{s \sinh (\omega T)}\right),
$$


with $f_{\mu}$ as in (5.46), and where $P$ is the following polynomial of $\mathcal{Z}_{1}, \ldots, \mathcal{Z}_{k}$

$$
\begin{aligned}
& P\left(\mathcal{Z}_{1}, \ldots, \mathcal{Z}_{k} \mid t_{1}, \ldots, t_{k}\right)=2 \cosh (\omega T) \\
& \quad+\sum_{r=1}^{k}(-1)^{r} \sum_{1 \leq m_{1}<m_{2}<\ldots<m_{r} \leq k} \mathcal{Z}_{m_{1}} \mathcal{Z}_{m_{2}} \ldots \mathcal{Z}_{m_{k}} \prod_{j=1}^{r} 2 \sinh \left(\omega\left(\sum_{m_{j} \leq s \leq m_{j+1}-1} t_{s}\right)\right),
\end{aligned}
$$

with suitable boundary conditions on the indices, namely: $m_{r+1} \equiv m_{1}+k$ and $t_{s} \equiv t_{s-k}$ for $s>k$. Note the remarkable fact that all the $k$-point correlators are expressed in terms of the same universal scaling function $g_{\mu}$ containing all the $\mu$-dependence.

Moreover, the results of this section clearly only depend on the parameters $\mu, \omega, T$, so forgetting about the dependence of $\mu$ and $\omega$ on $\beta$, we may in particular interpret the cases $\mu=1 / 2$ and $\mu=3 / 2$ in terms of a one-dimensional harmonic oscillator. Indeed, when $\mu=1 / 2$ (resp. $\mu=3 / 2$ ), our quantum system reduces to the even (resp. odd) sector of an ordinary harmonic oscillator with frequency $\omega$ on a time circle of length $T$. More precisely, the eigenvalues $E_{n}=\omega(2 n+\mu)$ (5.16) and eigenvectors $\psi_{n}$ (5.12) reduce respectively to

$$
\begin{aligned}
& \mu=\frac{1}{2}: \quad E_{n}=\omega\left(2 n+\frac{1}{2}\right), \quad \psi_{n}(\varphi)=\frac{(-1)^{n}}{2^{n-\frac{1}{2}} \sqrt{(2 n) !}\left(\frac{\omega}{\pi}\right)^{\frac{1}{4}} H_{2 n}(\sqrt{\omega} \varphi) e^{-\frac{1}{2} \omega \varphi^{2}},} \\
& \mu=\frac{3}{2}: \quad E_{n}=\omega\left(2 n+1+\frac{1}{2}\right), \quad \psi_{n}(\varphi)=\frac{(-1)^{n}}{2^{n} \sqrt{(2 n+1) !}}\left(\frac{\omega}{\pi}\right)^{\frac{1}{4}} H_{2 n+1}(\sqrt{\omega} \varphi) e^{-\frac{1}{2} \omega \varphi^{2}},
\end{aligned}
$$

in terms of the Hermite polynomials $H_{n}(x)$. Note that $\varphi$ is still restricted to be positive, but we may relax this condition by remarking that the Hermite polynomials of even degree are even, while the odd degree ones are odd. So we may extend the range of $\varphi$ to the whole real line, upon simply redefining the eigenvectors above $\psi_{n} \rightarrow \psi_{n} / \sqrt{2}$, and considering only even observables, say. The corresponding partition functions read respectively

$$
Z_{T, \frac{1}{2}}=\frac{e^{-\frac{\omega T}{2}}}{1-e^{-2 \omega T}} \quad \text { and } \quad Z_{T, \frac{3}{2}}=\frac{e^{-3 \frac{\omega T}{2}}}{1-e^{-2 \omega T}}
$$

and their sum is nothing but the partition function of the harmonic oscillator

$$
Z_{T, \mathrm{osc}}=Z_{T, \frac{1}{2}}+Z_{T, \frac{3}{2}}=\frac{e^{-\frac{\omega T}{2}}}{1-e^{-\omega T}}
$$

More generally, the result (5.49) for the correlation functions of the operator $e^{\omega \mathcal{N Z}}$ can be used to derive the corresponding quantity for the harmonic oscillator. Indeed, the odd 
and even sectors are orthogonal, and the full correlator reads

$$
\begin{aligned}
& \left\langle\prod_{m=1}^{k} e^{\omega \varphi^{2}\left(u_{m}\right) \mathcal{Z}_{m}}\right\rangle_{\mathrm{osc}}=\frac{1}{Z_{T, \mathrm{osc}}}\left(Z_{T, \frac{1}{2}} g_{\frac{1}{2}}(s)+Z_{T, \frac{3}{2}} g_{\frac{3}{2}}(s)\right) \\
& \quad=\sinh \left(\frac{\omega T}{2}\right) \sqrt{\frac{s}{1-s^{2}}}\left(s\left(1-\sqrt{1-s^{2}}\right)^{-\frac{1}{2}}+\left(1-\sqrt{1-s^{2}}\right)^{\frac{1}{2}}\right) \\
& \quad=\sinh \left(\frac{\omega T}{2}\right) \sqrt{\frac{2 s}{1-s}},
\end{aligned}
$$

where $s=2 / P\left(\mathcal{Z}_{1}, \ldots, \mathcal{Z}_{k} \mid t_{1}, \ldots, t_{k}\right)$ and $g_{\mu}$ as in (5.50). From (5.55) we learn that the corresponding correlation function in the harmonic oscillator case also only depends on the arguments of the observables through the quantity s. In Appendix C, we compute directly the correlator (5.55) within the framework of the harmonic oscillator and obtain an alternative expression for the combined argument $s$, which leads to the following determinantal expression for the polynomial $P$

$$
P\left(\mathcal{Z}_{1}, \ldots, \mathcal{Z}_{k} \mid t_{1}, \ldots, t_{k}\right)=2\left(1+2 \sinh ^{2}\left(\frac{\omega T}{2}\right) \frac{\operatorname{det}(M-Z)}{\operatorname{det}(M)}\right),
$$

where $M$ and $Z$ are the following $k \times k$ matrices

$$
M=\left(\begin{array}{ccccc}
\gamma_{1} & -x_{1} & 0 & \ldots & -x_{k} \\
-x_{1} & \gamma_{2} & -x_{2} & \ldots & 0 \\
0 & -x_{2} & \gamma_{3} & \ldots & \vdots \\
\vdots & \ddots & \vdots & \ddots & \vdots \\
-x_{k} & 0 & \cdots & -x_{k-1} & \gamma_{k}
\end{array}\right) \quad Z=\left(\begin{array}{ccccc}
\mathcal{Z}_{1} & 0 & 0 & \cdots & 0 \\
0 & \mathcal{Z}_{2} & 0 & \cdots & 0 \\
0 & 0 & \mathcal{Z}_{3} & \cdots & \vdots \\
\vdots & \ddots & \vdots & \ddots & \vdots \\
0 & \cdots & \cdots & 0 & \mathcal{Z}_{k}
\end{array}\right)
$$

with $x_{i}=1 /\left(2 \sinh \left(\omega t_{i}\right)\right)$, and $\gamma_{i}=2 x_{i} x_{i-1} \sinh \left(\omega\left(t_{i}+t_{i-1}\right)\right), i=1,2, \ldots, k$, with the convention $x_{0} \equiv x_{k}, t_{0} \equiv t_{k}$.

\section{Discussion}

\subsection{Lorentzian vs. Euclidean triangulations}

With the present work we have added yet another case to the list of problems for which the combination of methods from quantum field theory and statistical mechanics has proven very powerful (see for instance [1], 14]).

We have seen that in a certain region of the coupling constant space of our model, more precisely for $\beta<1$, it is possible to define a continuum limit. The coupling constant 
$\beta$ survives this limit in unrenormalized form and we get a one-parameter family of continuum models which are neither identical to the continuum description of pure Lorentzian triangulations nor to that of Euclidean ones. The models do, however, have some common features with the pure Lorentzian triangulation model: they have the same scaling of the time variable and thus the same fractal dimension $d_{H}=2$ of the continuum space-time manifolds. One could have hoped, bearing in mind our original aim at gaining a better understanding of the renormalization idea of [6], that one would get out Euclidean quantum gravity for $\beta=1$. However, we are led to the conclusion that no continuum limit exists for $\beta \geq 1$. The class of outgrowths that we considered here is too restricted to allow the model to flow from the Lorentzian case to the Euclidean one. What happens instead as $\beta$ increases from zero to one is that the correlation length $\xi$ defined by (cf. eqn. (5.17))

$$
Z_{T} \sim \exp \left(-\frac{1}{\xi} T\right), \quad \text { as } \quad \mathrm{T} \rightarrow \infty
$$

i.e.

$$
\xi=\left(\frac{1-\beta^{2}}{1+\beta+\beta^{2}}\right) \frac{1}{\sqrt{\Lambda}},
$$

decreases from $\frac{1}{\sqrt{\Lambda}}$ to zero. This means that our time slices effectively decouple from each other, not allowing us to interpret our model as a model of surfaces. This decoupling effect is of course due to the fact that the outgrowths which dominate the geometries more and more as $\beta$ increases do not imply any interaction between different time slices.

\subsection{Effective integrable structure and Renormalization Group formulation}

In ref. [9], a two-parameter family of theories has already been introduced to describe Lorentzian triangulations with intrinsic curvature energy (the parameters being the weight per triangle and a curvature weight). These models were found to be integrable, in the sense that their transfer matrices taken at different values of the curvature weight and of the weight per triangle but along the same spectral curve commute with one another. However, all these models turned out to share the same continuum limit up to a redefinition of the space and time scales which absorbed the (irrelevant) curvature parameter. One of those models is precisely the pure case $\beta=0$.

Here we have a very different situation: the parameter $\beta$ survives and leads to distinct continuous theories. On the other hand, the model we started from does not seem to be part of an integrable family (except for $\beta=0$ ). Still, the very simple form of the energies (5.16) suggests that for each value of $\beta<1$ there exists a two-parameter family of transfer 
matrices which is integrable and leads to the same continuum limit as our original model. The corresponding kernels are readily constructed by use of the quadratic relation (5.39). Upon taking

$$
z=\lambda, \quad \varphi_{0}=\sqrt{\alpha}, \varphi_{T}=\sqrt{\alpha^{\prime}}
$$

we may introduce the kernel

$$
\begin{aligned}
G_{\Omega, \lambda}\left(\alpha, \alpha^{\prime}\right) & \equiv \sum_{n \geq 0} \frac{\psi_{n}^{(\Omega)}(\sqrt{\alpha})}{\sqrt{2 \sqrt{\alpha}}} \frac{\psi_{n}^{(\Omega)}\left(\sqrt{\alpha^{\prime}}\right)}{\sqrt{2 \sqrt{\alpha^{\prime}}}} \lambda^{n+\frac{\mu}{2}} \\
& =\frac{\Omega \sqrt{\lambda}}{1-\lambda} e^{-\frac{1}{2} \frac{1+\lambda}{1-\lambda} \Omega\left(\alpha+\alpha^{\prime}\right)} I_{\mu-1}\left(2 \Omega \frac{\sqrt{\lambda}}{1-\lambda} \sqrt{\alpha \alpha^{\prime}}\right),
\end{aligned}
$$

where $\mu-1=\beta /\left(1+\beta^{2}\right)$ as before and the $\psi_{n}^{(\Omega)}$ are given by (5.12) with the substitution $\omega \rightarrow \Omega$. By construction, it is clear that

$$
\int_{0}^{\infty} d \alpha^{\prime \prime} G_{\Omega, \lambda}\left(\alpha, \alpha^{\prime \prime}\right) G_{\Omega, \lambda^{\prime}}\left(\alpha^{\prime \prime}, \alpha^{\prime}\right)=G_{\Omega, \lambda \lambda^{\prime}}\left(\alpha, \alpha^{\prime}\right)
$$

hence all the transfer kernels commute at fixed $\Omega$. Choosing

$$
\Omega=\Omega(\lambda) \equiv \frac{1-\lambda}{1+\lambda}\left(\frac{1-\beta^{2}}{1+\beta^{2}}\right)^{2}
$$

and rescaling the $\alpha$ 's as

$$
\tilde{\alpha}=\left(\frac{1-\beta^{2}}{1+\beta^{2}}\right)^{2} \alpha, \quad \tilde{\alpha}^{\prime}=\left(\frac{1-\beta^{2}}{1+\beta^{2}}\right)^{2} \alpha^{\prime}
$$

we get a one-parameter family of kernels

$$
\tilde{G}_{\lambda}\left(\tilde{\alpha}, \tilde{\alpha}^{\prime}\right) \equiv\left(\frac{1+\beta^{2}}{1-\beta^{2}}\right)^{2} G_{\Omega(\lambda), \lambda}\left(\alpha, \alpha^{\prime}\right)=\frac{\sqrt{\lambda}}{1+\lambda} e^{-\frac{1}{2}\left(\tilde{\alpha}+\tilde{\alpha}^{\prime}\right)} I_{\mu-1}\left(2 \frac{\sqrt{\lambda}}{1+\lambda} \sqrt{\tilde{\alpha} \tilde{\alpha}^{\prime}}\right)
$$

The kernels (6.8) lead to the same continuum limit $a \rightarrow 0$ as our original generalized Lorentzian triangulation model with parameters $\beta$ and $g \theta$ upon taking

$$
\lambda=e^{-2 a \omega}=e^{-2 a \sqrt{\Lambda}\left(\frac{1+\beta^{2}}{1-\beta^{2}}\right)},
$$

with the same $a$ and $\Lambda$ as in (4.24). 
We may now reinterpret the composition formula (6.5) as a self-similarity property for the kernel $\tilde{G}_{\lambda}$. Indeed, taking $\lambda=\lambda^{\prime}$, we get

$$
\begin{aligned}
\sqrt{d \tilde{\alpha} d \tilde{\alpha}^{\prime}} \int_{0}^{\infty} d \tilde{\alpha}^{\prime \prime} \tilde{G}_{\lambda}\left(\tilde{\alpha}, \tilde{\alpha}^{\prime \prime}\right) \tilde{G}_{\lambda}\left(\tilde{\alpha}^{\prime \prime}, \tilde{\alpha}^{\prime}\right)=\sqrt{d \alpha d \alpha^{\prime}} G_{\Omega(\lambda), \lambda^{2}}\left(\alpha, \alpha^{\prime}\right) \\
=\frac{\lambda}{(1+\lambda)^{2}} \sqrt{d \tilde{\alpha} d \tilde{\alpha}^{\prime}} e^{-\frac{1}{2} \frac{1+\lambda^{2}}{(1+\lambda)^{2}}\left(\tilde{\alpha}+\tilde{\alpha}^{\prime}\right)} I_{\mu-1}\left(2 \frac{\lambda}{(1+\lambda)^{2}} \sqrt{\tilde{\alpha} \tilde{\alpha}^{\prime}}\right) \\
=\frac{\lambda}{1+\lambda^{2}} \sqrt{d \hat{\alpha} d \hat{\alpha}^{\prime}} e^{-\frac{1}{2}\left(\hat{\alpha}+\hat{\alpha}^{\prime}\right)} I_{\mu-1}\left(2 \frac{\lambda}{1+\lambda^{2}} \sqrt{\hat{\alpha} \hat{\alpha}^{\prime}}\right) \\
=\sqrt{d \hat{\alpha} d \hat{\alpha}^{\prime}} \tilde{G}_{\lambda^{2}}\left(\hat{\alpha}, \hat{\alpha}^{\prime}\right),
\end{aligned}
$$

where we have performed the change of variables $\hat{\alpha}=\frac{1+\lambda^{2}}{(1+\lambda)^{2}} \tilde{\alpha}$ and similarly on $\alpha^{\prime}$, and as before $\tilde{\alpha}=\left(\frac{1-\beta^{2}}{1+\beta^{2}}\right)^{2} \alpha$. The operation performed in 6.10 decomposes into two steps: (i) composition of $\tilde{G}_{\lambda}$ with itself, by integration over the intermediate variable $\tilde{\alpha}^{\prime \prime}$ (ii) rescaling of the variables $\tilde{\alpha}, \tilde{\alpha}^{\prime} \rightarrow \hat{\alpha} \hat{\alpha}^{\prime}$, to yield the kernel $\tilde{G}_{\lambda^{2}}$. This is nothing but a real-space functional renormalization group $(\mathrm{RG})$ decimation procedure. Indeed, imagine we wish to evaluate a product of say $2^{n}$ identical kernels $\tilde{G}_{\lambda}$. We first apply (6.10) to the $2^{n-1}$ consecutive pairs of kernels, and end up after rescaling with a product of $2^{n-1}$ kernels $\tilde{G}_{\lambda^{2}}$, with the renormalized value $\lambda^{2}$ of the parameter. Iterating the process will lead to the final single kernel $\tilde{G}_{\lambda^{2}}\left(\alpha_{n}, \alpha_{n}^{\prime}\right)$, where

$$
\alpha_{n}=\frac{1+\lambda^{2^{n}}}{\left(1+\lambda^{2^{n-1}}\right)^{2}} \alpha_{n-1}, \quad \alpha_{0}=\tilde{\alpha}
$$

and similarly for $\alpha_{n}^{\prime}$. This is easily solved as

$$
\alpha_{n}=\frac{1+\lambda^{2^{n}}}{1-\lambda^{2^{n}}} \frac{1-\lambda}{1+\lambda} \tilde{\alpha}
$$

and similarly for $\alpha_{n}^{\prime}$. This yields an alternative derivation of the loop-loop correlator (5.35) over a time lapse $t=2^{n}$. Setting $\lambda=e^{-2 a \omega}, t=2^{n}=T / a$ as usual and taking the initial boundary values

$$
\alpha_{0}=\tilde{\alpha}=2\left(\frac{1-\beta^{2}}{1+\beta^{2}}\right)^{2} \frac{L_{0}}{a}, \quad \alpha_{0}^{\prime}=\tilde{\alpha}^{\prime}=2\left(\frac{1-\beta^{2}}{1+\beta^{2}}\right)^{2} \frac{L_{T}}{a},
$$

we get the iterated values

$$
\alpha_{n}=2 \omega \operatorname{cotanh}(\omega T)\left(\frac{1-\beta^{2}}{1+\beta^{2}}\right)^{2} L_{0}, \quad \alpha_{n}^{\prime}=2 \omega \operatorname{cotanh}(\omega T)\left(\frac{1-\beta^{2}}{1+\beta^{2}}\right)^{2} L_{T},
$$


so that the loop-loop correlator reads

$$
\begin{aligned}
& Z_{T}\left(L_{0}, L_{T}\right)=2\left(\frac{1-\beta^{2}}{1+\beta^{2}}\right)^{2} \omega \operatorname{cotanh}(\omega T) \times \\
& \tilde{G}_{e^{-2 \omega T}}\left(2 \omega \operatorname{cotanh}(\omega T)\left(\frac{1-\beta^{2}}{1+\beta^{2}}\right)^{2} L_{0}, 2 \omega \operatorname{cotanh}(\omega T)\left(\frac{1-\beta^{2}}{1+\beta^{2}}\right)^{2} L_{T}\right),
\end{aligned}
$$

which is nothing but (5.40).

The self-similar kernels under RG transformations have been studied and classified in ref. [15] in the context of 1+1-dimensional critical wetting, and correspond precisely to the form (6.8) above. Each universality class is entirely characterized by the value of $\mu-1=\beta /\left(1+\beta^{2}\right)$. Our original model for decorated Lorentzian triangulations provides therefore an explicit discrete and simple realization of these universality classes.

\subsection{Calogero vs random walks}

For $\beta<1$ where a continuum limit exists our generalized Lorentzian triangulation model is equivalent to a one-dimensional Calogero model. This model is known to appear in many different physical problems. It would be interesting to understand whether our generalized Lorentzian triangulation model thus has an interpretation in terms of a completely different physical system. One particular realization of such a different equivalent physical system has been found in [9] where the pure Lorentzian case $\beta=0$ was shown to be equivalent to a one-dimensional Random Walk (RW) confined in a segment of size $t$ upon identifying the fugacity per triangle $g$ with the fugacity per step of walk. The RW model is known to be expressible as a massive scalar field theory in one dimension with action

$$
\mathcal{S}(\varphi)=\frac{1}{2} \int d u\left(\varphi^{\prime}(u)^{2}+\omega^{2} \varphi(u)^{2}\right),
$$

which is nothing but the ordinary quantum theory of a harmonic oscillator. The quantity

$\varphi(u)^{2}$ is interpreted as the density of RW at point $u$, which precisely translates into the quantity $\mathcal{N}(u)$ (3.27) under the abovementioned equivalence, while $\omega$ is the continuous counterpart of the fugacity per step of walk.

Surprisingly enough, we have stumbled here on the one-dimensional Calogero model at $A=1$, rather than the harmonic oscillator corresponding to the action (6.16) above. 


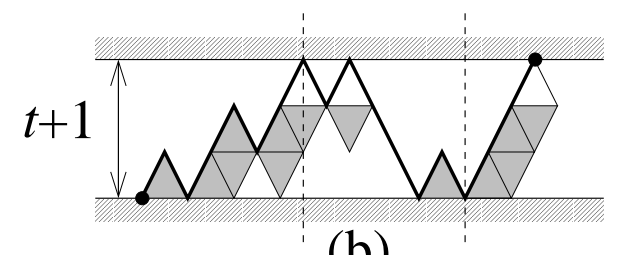

(b)

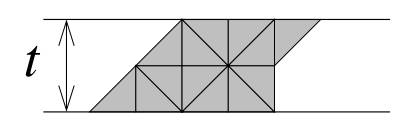

(a)
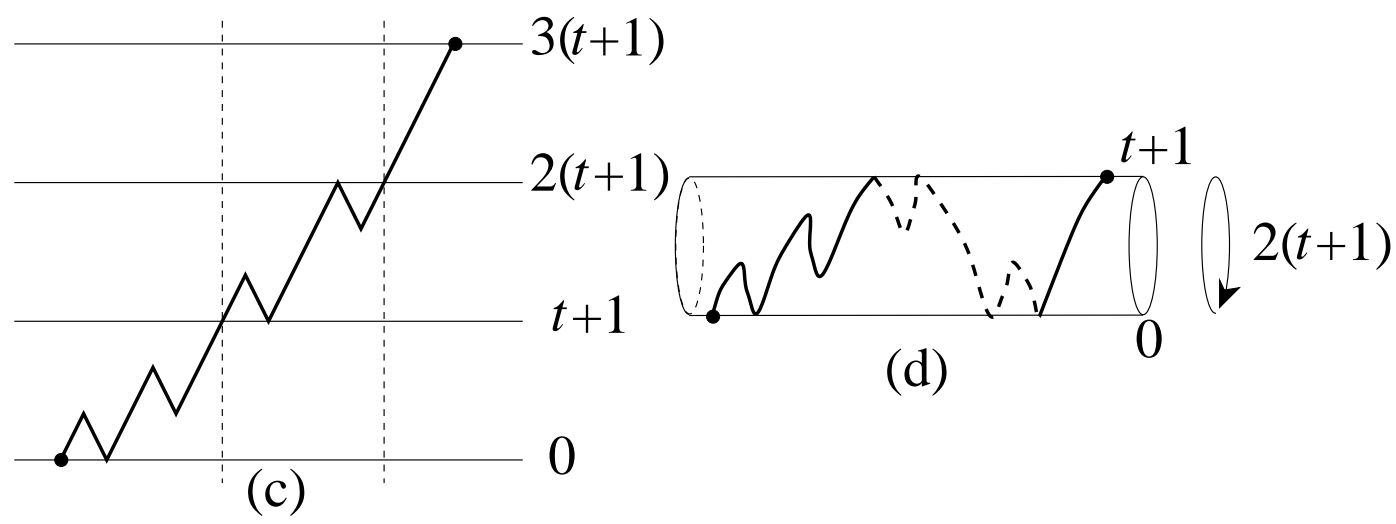

(d)

Fig. 5: Random Walk picture of pure Lorentzian triangulations. A Lorentzian triangulation (a) of time size $t$ and its representation as a RW (b) between two reflecting walls at distance $t+1$ (to go from (b) to (a), simply squeeze the triangles horizontally). The reflected walk can be unraveled (c), each portion between two contacts being reflected or not (here we made for instance two reflections at positions indicated by the dashed vertical lines. We finally compactify the time direction onto a circle of perimeter $2(t+1)$, ending with a RW (d) attached at the antipodal points 0 and $t+1$.

Note that due to the extra potential $1 /\left(8 \varphi^{2}\right)$, a crucial difference is that the range of $\varphi$ is over $\mathbb{R}_{+}$instead of $\mathbb{R}$ for the harmonic case.

It is an interesting exercise to re-derive the pure Lorentzian triangulation results in the RW harmonic oscillator language. In the following we will concentrate on the loop-loop correlator $Z(i, j, t)$ defined in (5.18). In ref. [9], it was shown that

$$
Z_{i, j}(t) \equiv g^{t} Z(i-1, j-1, t)=\frac{1}{(2 g)^{i+j-1}} \sum_{S \geq 0}(2 g)^{S} P(i, j, S ; t+1)
$$

where $P(i, j, S ; t+1)$ is the probability for a walker on the discrete integer segment $[0, t+$ 1] $\subset \mathbb{N}$ making steps of \pm 1 with probability $1 / 2$ at any integer position $0<h<t+1$ and being reflected with probability 1 whenever it reaches the boundaries $h=0$ or $t+1$, to go from the position $h=0$ to the position $h=t+1$ in $S$ steps, with exactly $i$ visits at $h=0$ and $j$ visits at $h=t+1$ (see Fig. 5). The slightly different transfer matrix $g^{i+j-1}\left(\begin{array}{c}i+j-2 \\ i-1\end{array}\right)$ used in [9] is responsible for the the shifts by -1 of $i$ and $j$, and for the factor $g$ per time step. 
The reflection condition at $h=0$ and $t+1$ may be unraveled by associating to each walk on $[0, t+1]$ the set of walks on $\mathbb{Z}$ obtained by iteratively reflecting or not each portion between two contacts at the boundaries (see Fig. 5) These new walks now go from the position $h=0$ to some position $h=(2 k+1)(t+1)$ with $k \in \mathbb{Z}$. The numbers of original contacts $i$ and $j$ with respectively the boundaries $h=0$ and $h=t+1$ translate into the total numbers of contacts with respectively the lines $h=2 k(t+1)$ and $h=(2 k+1)(t+1)$, $k \in \mathbb{Z}$. By compactifying the target space $\mathbb{Z}$ onto the circle $\mathbb{Z} /(2(t+1) \mathbb{Z})$, we end up with walks from $h=0$ to $h=t+1$ on a circle of perimeter $2(t+1)$ as shown in Fig. 5 . In this final picture, $i$ and $j$ represent respectively the numbers of visits of the antipodal positions $h=0$ and $h=t+1$.

In the continuum limit $t=T / a, g=\frac{1}{2}\left(1-a^{2} \frac{\omega^{2}}{2}\right)$, the latter RW's are governed by an action of the form (6.16) with $u \in[0,2 T]$ and the cyclic boundary condition $\varphi(u+2 T)=$ $\varphi(u)$. In this language the rescaled numbers of visits ai and aj become respectively $\varphi(0)^{2}$ and $\varphi(T)^{2}$. Moreover, it is simpler to compute the rescaled loop-loop correlator (5.22) with $\beta=0$, by weighting each visit at $h=0$ and $t+1$ respectively by $x$ and $y$ and setting $x=1-a X, y=1-a Y$ as before. We end up with the equivalence

$$
Z_{T}(X, Y)=\frac{\left\langle 2 \varphi(0) \varphi(T) e^{-X \varphi(0)^{2}} e^{-Y \varphi(T)^{2}}\right\rangle}{\left\langle e^{-X \varphi(0)^{2}} e^{-Y \varphi(T)^{2}}\right\rangle}
$$

where $\langle\ldots\rangle$ stands for the functional average using the abovementioned action for the harmonic oscillator on a circle of perimeter $2 T$. In eqn.(6.18), the factors $\varphi(0)$ and $\varphi(T)$ ensure that the walk starts at 0 and ends at $T$ (the factor 2 relates to our choice of normalization of the action). The insertions of exponential terms account precisely for the weights $x, y$ per visit at $0, t+1$, and the denominator ensures the suppression of the contribution of disconnected walks. It is now a simple exercise in $1 \mathrm{D}$ quantum mechanics to evaluate the rhs of (6.18) namely, using the harmonic oscillator Hamiltonian $H_{\mathrm{osc}}=-\frac{1}{2} \frac{d^{2}}{d \varphi^{2}}+\frac{1}{2} \omega^{2} \varphi^{2}$ :

$$
Z_{T}(X, Y)=\frac{\int_{-\infty}^{\infty} 2 \varphi_{0} d \varphi_{0} \varphi_{T} d \varphi_{T} e^{-\left(\varphi_{0}^{2} X+\varphi_{T}^{2} Y\right)}\left(\left\langle\varphi_{T}\left|e^{-T H_{\mathrm{osc}}}\right| \varphi_{0}\right\rangle\right)^{2}}{\int_{-\infty}^{\infty} d \varphi_{0} d \varphi_{T} e^{-\left(\varphi_{0}^{2} X+\varphi_{T}^{2} Y\right)}\left(\left\langle\varphi_{T}\left|e^{-T H_{\mathrm{osc}}}\right| \varphi_{0}\right\rangle\right)^{2}}
$$

to be compared with the Calogero formulation (5.23). Using the diagonalization formula (C.4) of appendix $\mathrm{C}$ below, with $z=e^{-\omega T}$, we are left with a ratio of two two-dimensional Gaussian integrals

$$
Z_{T}(X, Y)=\frac{\int_{\mathbb{R}^{2}} d^{2} \vec{\varphi} 2 \varphi_{1} \varphi_{2} e^{-\frac{1}{2} \vec{\varphi} \cdot M \vec{\varphi}}}{\int_{\mathbb{R}^{2}} d^{2} \vec{\varphi} e^{-\frac{1}{2} \vec{\varphi} \cdot M \vec{\varphi}}}=2\left(M^{-1}\right)_{1,2}
$$


with the matrix

$$
M=2\left(\begin{array}{cc}
X+\omega \operatorname{cotanh}(T \omega) & -\frac{\omega}{\sinh (T \omega)} \\
-\frac{\omega}{\sinh (T \omega)} & Y+\omega \operatorname{cotanh}(T \omega)
\end{array}\right)
$$

leading finally to the previous result (5.33), with $\omega=\sqrt{\Lambda}$.

In view of the above derivation, it is remarkable that the Calogero formulation of the problem automatically takes care of all the subtleties (boundary conditions, suppression of disconnected loops, etc ...) we encountered in the RW approach. In this regard, the Calogero formulation of pure Lorentzian triangulations is better adapted.

\subsection{Calogero vs 2D harmonic oscillator}

The previous section is a manifestation of the well-known connection between the Calogero Hamiltonian at $\mu=1$ and the harmonic oscillator. As we will see now, the Schwinger representation we have developed in this paper provides another direct and exact link between the pure model and a pair of (untwisted/twisted) harmonic oscillators. This connection holds even before taking the continuum limit.

Let us start again from the $\beta=0, h=1$ counterpart of (3.7) in which we also have incorporated for convenience an entropic factor $g$ per time slice, namely

$$
g^{t} Z^{(0)}(t)=\prod_{s=1}^{t}\left(\oint_{\mathcal{E}} \frac{d \rho_{s}}{2 i \pi \rho_{s}} \int_{0}^{\infty} d \alpha_{s} e^{-\alpha_{s}}\right) \prod_{r=1}^{t} g e^{g \alpha_{r}\left(\rho_{r}+\frac{1}{\rho_{r-1}}\right)}, \quad \rho_{0}=\rho_{t} .
$$

We are about to show that the coordinates $\alpha>0$ and $\rho \in \mathcal{E}$ may be rephrased into polar coordinates of the real plane, pertaining themselves to two one-dimensional harmonic oscillators. Performing the change of variable

$$
\begin{aligned}
& \rho_{r}=\sqrt{\frac{\alpha_{r+1}}{\alpha_{r}}} e^{i \chi_{r+1}}, \quad r=0,1, \ldots, t-1, \\
& \alpha_{r}=R_{r}^{2}, \quad r=0,1, \ldots, t
\end{aligned}
$$

with $\alpha_{0} \equiv \alpha_{t}$ and where we have deformed the contours so as to have all $\chi_{r} \in[0,2 \pi)$, we get

$$
g^{t} Z^{(0)}(t)=\prod_{s=1}^{t}\left(\int_{0}^{2 \pi} \frac{d \chi_{s}}{2 \pi} \int_{0}^{\infty} 2 R_{s} d R_{s} e^{-R_{s}^{2}}\right) \prod_{r=1}^{t} g e^{2 g R_{r} R_{r-1} \cos \left(\chi_{r}\right)} .
$$


Finally, let us perform the following changes of variables on the $\chi$ 's, according to the parity of $t$ :

$$
\begin{aligned}
t \text { odd }: & \chi_{r}=\left\{\begin{array}{cc}
\theta_{r}+\theta_{r-1}, & r=2,3, \ldots, t \\
\theta_{1}+\theta_{t}, & r=1
\end{array}\right. \\
t \text { even }: & \chi_{r}=\left\{\begin{array}{cc}
\theta_{r}+\theta_{r-1}, & r=2,3, \ldots, t \\
\theta_{1}-\theta_{t}, & r=1
\end{array}\right.
\end{aligned}
$$

with $\theta_{r} \in[0,2 \pi), r=1,2, \ldots, t$. The extra sign in the case $t$ even is ad-hoc to make the change of variables invertible. We can now interpret

$$
\left(x_{r}=R_{r} \cos \theta_{r}, y_{r}=R_{r} \sin \theta_{r}\right),
$$

as two real coordinates of the plane $\mathbb{R}^{2}$, so that (6.24) becomes

$$
g^{t} Z^{(0)}(t)=\prod_{s=1}^{t}\left(\frac{1}{\pi} \int_{\mathbb{R}^{2}} d x_{s} d y_{s} e^{-\left(x_{s}^{2}+y_{s}^{2}\right)}\right) g e^{2 g\left(x_{1} x_{t}-(-1)^{t-1} y_{1} y_{t}\right)} \prod_{r=2}^{t} g e^{2 g\left(x_{r-1} x_{r}-y_{r-1} y_{r}\right)} .
$$

This last formula suggests to introduce the following transfer kernel:

$$
G_{g}\left(x, y ; x^{\prime}, y^{\prime}\right)=\frac{g}{\pi} e^{-\frac{1}{2}\left(x^{2}+y^{2}+x^{\prime 2}+y^{\prime 2}\right)} e^{2 g\left(x x^{\prime}-y y^{\prime}\right)},
$$

in terms of which the partition function reads:

$$
g^{t} Z^{(0)}(t)=\prod_{s=1}^{t}\left(\int_{\mathbb{R}^{2}} d x_{s} d y_{s}\right) G_{g}\left(x_{t},(-1)^{t-1} y_{t} ; x_{1}, y_{1}\right) \prod_{r=2}^{t} G_{g}\left(x_{r-1}, y_{r-1} ; x_{r}, y_{r}\right) .
$$

The transfer kernel (6.28) factorizes into $G_{g}\left(x, y ; x^{\prime}, y^{\prime}\right)=G_{g}^{+}\left(x, x^{\prime}\right) G_{g}^{-}\left(y, y^{\prime}\right)$, where the two kernels $G_{g}^{ \pm}$respectively correspond to the harmonic oscillator and its twisted version, namely

$$
\begin{aligned}
& G_{g}^{+}\left(x, x^{\prime}\right)=\frac{\sqrt{g}}{\sqrt{\pi}} e^{-\frac{1}{2}\left(x^{2}+x^{\prime 2}-4 g x x^{\prime}\right)}=\sum_{n \geq 0} \psi_{n}^{\mathrm{osc}}(x) \psi_{n}^{\mathrm{osc}}\left(x^{\prime}\right) q^{n+\frac{1}{2}} \\
& G_{g}^{-}\left(y, y^{\prime}\right)=\frac{\sqrt{g}}{\sqrt{\pi}} e^{-\frac{1}{2}\left(y^{2}+y^{\prime 2}+4 g y y^{\prime}\right)}=\sum_{n \geq 0}(-1)^{n} \psi_{n}^{\mathrm{osc}}(y) \psi_{n}^{\mathrm{osc}}\left(y^{\prime}\right) q^{n+\frac{1}{2}},
\end{aligned}
$$

where we have set

$$
g=\frac{1}{q+\frac{1}{q}}
$$

and the $n$-th eigenfunction of the harmonic oscillator reads

$$
\psi_{n}^{\mathrm{osc}}(\varphi)=\sqrt{\frac{\sqrt{\omega}}{\sqrt{\pi} 2^{n} n !}} H_{n}(\sqrt{\omega} \varphi) e^{-\frac{1}{2} \omega \varphi^{2}}
$$


where $H_{n}(x)$ are the Hermite polynomials. The factorization of the kernel implies that of the partition function

$$
g^{t} Z^{(0)}(t)=Z_{+}^{\mathrm{osc}}(t) Z_{-}^{\mathrm{osc}}(t)
$$

where, writing formally products of kernels like ordinary products, we have

$$
Z_{+}^{\mathrm{osc}}(t)=\operatorname{Tr}\left(\left(G_{g}^{+}\right)^{t}\right)=\frac{q^{\frac{t}{2}}}{1-q^{t}}, \quad Z_{-}^{\mathrm{osc}}(t)=\operatorname{Tr}\left(\left(G_{g}^{-}\right)^{t} S^{\frac{1+(-1)^{t}}{2}}\right)=\frac{q^{\frac{t}{2}}}{1+q^{t}}
$$

where $S$ has the kernel $S\left(y, y^{\prime}\right)=\delta\left(y+y^{\prime}\right)$. Substituting these into (6.33) yields (3.19). When we take the continuum limit $a \rightarrow 0$ (3.23), both $Z_{+}^{\text {osc }}$ and $Z_{-}^{\text {osc }}$ become functional integrals of a scalar free field with action (6.16), the only difference being the boundary condition, namely $\varphi(u+T)=\varphi(u)$ for the oscillator and $\varphi(u+T)=-\varphi(u)$ for its twisted version. Indeed, in the latter case, we may perform the change of variables $y_{2 r} \rightarrow-y_{2 r}$ for the even indices, while the $y_{2 r+1}$ remain unchanged in order to recover the correct sign of the untwisted case, but for both parities of $t$ we end up with a wrong sign for the term $y_{1} y_{t}$, translating in the continuum limit into the above twisted boundary condition. The occurrence of both (twisted and untwisted) sectors arises from the fact that our boundary condition simply imposes that the rescaled number of triangles $\mathcal{N}$ at $u=0$ and $u=T$ are identical, namely $\varphi(u+T)^{2}=\varphi(u)^{2}$, which decomposes into periodic and antiperiodic boundary conditions for the free field itself. This factorization property extends to correlations involving the observables $\Sigma_{s}(z)(3.12)$, namely

$$
\begin{aligned}
\left\langle\prod_{m=1}^{k} \Sigma_{s_{m}}\left(z_{m}\right)\right\rangle_{\Theta} & =\left\langle\prod_{m=1}^{k} \frac{e^{\frac{\alpha_{s_{m}} z_{m}}{1+z_{m}}}}{1+z_{m}}\right\rangle_{G} \\
& =\left\langle\prod_{m=1}^{k} \frac{e^{\frac{x_{s_{m}}^{2} z_{m}}{1+z_{m}}}}{\sqrt{1+z_{m}}}\right\rangle_{G_{g}^{+}} \times\left\langle\prod_{m=1}^{k} \frac{e^{\frac{y_{s_{m}}^{2} z_{m}}{1+z_{m}}}}{\sqrt{1+z_{m}}}\right\rangle_{G_{g}^{-}} .
\end{aligned}
$$

This explains in particular the factorized form of the one-point average (3.22). Note that the above steps cannot be retraced for $\beta>0$, where no such free field formulation seems to exist.

It is instructive to re-derive the loop-loop correlator along the same lines as above. We start from

$$
g^{t+1} Z^{(0)}(x, y, t)=\prod_{s=1}^{t} \oint_{\mathcal{E}} \frac{d \rho_{s}}{2 i \pi \rho_{s}} \prod_{s=1}^{t+1} \int_{0}^{\infty} d \alpha_{s} e^{-\alpha_{s}} \prod_{r=1}^{t+1} g e^{g \alpha_{r}\left(\rho_{r}+\frac{1}{\rho_{r-1}}\right)}, \quad \frac{1}{\rho_{0}}=x, \rho_{t+1}=y .
$$


Let us change variables as before to $\alpha_{r}=R_{r}^{2}, r=1,2, \ldots, t+1, \rho_{r}=e^{i \chi_{r+1}} R_{r+1} / R_{r}, r=$ $1,2, \ldots, t$, and $\chi_{r}=\theta_{r}+\theta_{r-1}, r=2,3, \ldots, t+1$, where we choose $\theta_{1}$ arbitrarily. We end up with $t+1$ two-dimensional integrals over $\left(x_{r}, y_{r}\right)=\left(R_{r} \cos \theta_{r}, R_{r} \sin \theta_{r}\right), r=1,2, \ldots, t+1$, by adding for convenience an extra integral $\frac{1}{2 \pi} \int_{0}^{2 \pi} d \theta_{1}$, since the result does not depend on the choice $\theta_{1}$. This yields

$$
\begin{aligned}
& g^{t+1} Z^{(0)}(x, y, t)= \\
& \quad \prod_{s=1}^{t+1}\left(\frac{1}{\pi} \int_{\mathbb{R}^{2}} d x_{s} d y_{s} e^{-\left(x_{s}^{2}+y_{s}^{2}\right)}\right) g e^{g x\left(x_{1}^{2}+y_{1}^{2}\right)+g y\left(x_{t+1}^{2}+y_{t+1}^{2}\right)} \prod_{r=2}^{t+1} g e^{2 g\left(x_{r-1} x_{r}-y_{r-1} y_{r}\right)} .
\end{aligned}
$$

Note that if we switch to $y_{2 r} \rightarrow y_{2 r}$ and $y_{2 r-1} \rightarrow-y_{2 r-1}$, the integrals over the $x$ 's and the $y$ 's are identical, leading to

$$
\begin{aligned}
& g^{t+1} Z^{(0)}(x, y, t)=\operatorname{det}^{-1}\left(\begin{array}{ccccc}
\frac{1}{g}-x & -1 & 0 & \cdots & 0 \\
-1 & \frac{1}{g} & -1 & \ddots & \vdots \\
0 & \ddots & \ddots & \ddots & 0 \\
\vdots & \ddots & -1 & \frac{1}{g} & -1 \\
0 & \cdots & 0 & -1 & \frac{1}{g}-y
\end{array}\right) \\
& =\frac{q^{t}\left(1-q^{2}\right)}{(1-q x)(1-q y)-q^{2 t}(q-x)(q-y)} \text {, }
\end{aligned}
$$

where $\frac{1}{g}=q+\frac{1}{q}$, and the matrix is of size $(t+1) \times(t+1)$. This is in perfect agreement with the result of ref. [9] and with the continuum limit (5.33).

To conclude this section, we have unearthed an explicit two-component free field structure in the pure Lorentzian triangulation model, as opposed to the one-component free field description of Sect. 6.3 above. This increase in dimension is apparently the price to pay for reducing the interval $[0,2 T]$ of the latter description to the original one $[0, T]$. Indeed the information needed to reconstruct the observables at time $u$ pertains to the pair of slices $u$ and $2 T-u$ in the one-dimensional free field model.

This two-dimensional structure a posteriori explains the emergence of the $A=1$ Calogero model in its continuum description: indeed, the corresponding two-dimensional Schrödinger equation

$$
\left(-\frac{1}{2}\left(\partial_{x}^{2}+\partial_{y}^{2}\right)+\frac{1}{2} \omega^{2}\left(x^{2}+y^{2}\right)\right) \psi(x, y \mid t)=\partial_{t} \psi(x, y \mid t)
$$


when restricted to the rotation-invariant sector simply reads

$$
\left(-\frac{1}{2}\left(\partial_{R}^{2}+\frac{1}{R} \partial_{R}\right)+\frac{1}{2} \omega^{2} R^{2}\right) \psi(R \mid t)=\partial_{t} \psi(R \mid t)
$$

Upon redefining $\psi(R \mid t)=R^{-\frac{1}{2}} \phi(R \mid t)$, we end up with the Schrödinger equation with Calogero potential

$$
\left(-\frac{1}{2} \partial_{R}^{2}+\frac{1}{2} \omega^{2} R^{2}-\frac{1}{8 R^{2}}\right) \phi(R \mid t)=\partial_{t} \phi(R \mid t) .
$$

In this section, we have only considered radial observables thus ignoring completely the angular sector of the theory: it would be interesting to find the meaning of the angles in the triangulation language.

Finally, it would be interesting to understand if it is possible to construct a surface (or volume) model for which the continuum theory would belong to the class of higher dimensional (multi-particle) Calogero models which are known to have a much richer structure than the simple one, but this is another story.

\section{Acknowledgments:}

We thank J. Ambjørn, M. Bergère and T. Garel for useful discussions. All authors acknowledge support by the EU network on "Discrete Random Geometry", grant HPRNCT-1999-00161.

\section{Appendix A. An alternative analysis of the transfer matrix}

In this section we show how to recover the Calogero Hamiltonian with $A=1$ in the pure case using the strategy of ref. [3]. We also explain why the same strategy becomes much more involved for $\beta \neq 0$ and cannot apply to $\beta \geq 1$.

The starting point is the composition law [3]

$$
\Theta^{(1)}(x, y, t+1)=\oint_{\mathcal{C}} \frac{d \omega}{2 \pi i \omega} \Theta^{(1)}\left(x, \frac{1}{\omega}\right) \Theta^{(1)}(\omega, y, t),
$$

where $\Theta^{(1)}(x, y, t)=\left(\Theta^{(1)}\right)^{t}(x, y)$ with $\Theta^{(1)}(x, y)$ given by 2.5$)$. Here we can choose the contour $\mathcal{C}$ to encircle the singularities of $\frac{1}{\omega} \Theta^{(1)}\left(x, \frac{1}{\omega}\right)$, which consist of a simple pole, and not those of $\Theta^{(1)}(\omega, y, t)$. Inserting the scaling relations

$$
\begin{gathered}
g=\frac{1}{2}\left(1-\frac{1}{2} a^{2} \Lambda\right), \quad x=1-a X, \quad y=1-a Y, \quad \omega=1-a Z, \quad t=\frac{T}{a}, \\
\Theta^{(1)}(X, Y, T) \equiv \frac{a}{2^{t}} \Theta^{(1)}(x, y, t),
\end{gathered}
$$


and assuming the time evolution to be described in the scaling limit by a Hamiltonian $\hat{H}$, one gets for the evolution on an elementary time lapse $T=a$ :

$$
\begin{aligned}
\left(1-a \hat{H}+O\left(a^{2}\right)\right) \psi & =\frac{1}{2} \oint \frac{d \omega}{2 \pi i \omega} \Theta^{(1)}\left(x, \frac{1}{\omega}\right) \psi(\omega) \\
& =\int_{-i \infty+c}^{i \infty+c} \frac{d Z}{2 \pi i}\left\{\frac{1}{Z-X}+a\left(\frac{Z}{Z-X}+\frac{\Lambda-Z^{2}}{(Z-X)^{2}}\right)+O\left(a^{2}\right)\right\} \psi(Z),
\end{aligned}
$$

where $c$ is chosen so that the integration contour lies to the right of the pole at $Z=X$, and we have denoted by the same letter $\psi(\omega=1-a Z) \equiv \psi(Z)$. This gives

$$
\hat{H}=\left(X^{2}-\Lambda\right) \frac{\partial}{\partial X}+X
$$

Writing $\psi(X)=\int_{0}^{\infty} d L e^{-X L} \psi(L), L$ has the interpretation of the continuum loop length (cf. eqn. (2.3) ) and the inverse- Laplace transformed Hamiltonian $\hat{H}$ acting on functions of $L$ takes the form

$$
\hat{H}=-L \frac{\partial^{2}}{\partial L^{2}}-\frac{\partial}{\partial L}+\Lambda L
$$

with a flat measure on $L$ for the wave functions $\psi(L)$. Then performing the simultaneous change of variables and functions

$$
L=\frac{1}{2} \varphi^{2}, \quad \phi(\varphi)=\sqrt{\varphi} \psi\left(\frac{\varphi^{2}}{2}\right)
$$

guaranteeing a flat measure on $\varphi$ for $\phi(\varphi)$, we finally get

$$
\hat{H}=-\frac{1}{2} \frac{\partial^{2}}{\partial \varphi^{2}}+\frac{1}{2} \Lambda \varphi^{2}-\frac{1}{8} \frac{1}{\varphi^{2}}
$$

which coincides exactly with the result (5.10) for $\beta=0$.

Now, let us try to apply the same strategy for $\beta \neq 0$. Evidently, we need first to evaluate the transfer matrix. For the present calculation it proves convenient to consider its symmetrized version, i.e.

$$
\Theta(x, y)=\oint_{\mathcal{C}} \frac{d \omega_{1}}{2 \pi i \omega_{1}} \oint_{\mathcal{C}} \frac{d \omega_{2}}{2 \pi i \omega_{2}}\left(\Theta^{(1)}\right)^{1 / 2}\left(x, \frac{1}{\omega_{1}}\right) \Theta^{(2)}\left(\omega_{1}, \omega_{2}\right)\left(\Theta^{(1)}\right)^{1 / 2}\left(\frac{1}{\omega_{2}}, y\right) .
$$

Since the transfer matrix of the pure model has been explicitly diagonalized [9], it is straightforward to write down an expression for $\left(\Theta^{(1)}\right)^{1 / 2}(x, y)$

$$
\left(\Theta^{(1)}\right)^{1 / 2}(x, y)=\frac{C_{1}(x)}{1-C_{2}(x) y}=\frac{C_{1}(y)}{1-C_{2}(y) x},
$$


where

$$
C_{1}(x)=\frac{(1+2 g)^{1 / 2}}{1+g(1-x)}, \quad C_{2}(x)=\frac{g(1+x)}{1+g(1-x)} .
$$

Here we have chosen to write $\left(\Theta^{(1)}\right)^{1 / 2}(x, y)$ in a form which explicitly exposes its singularity structure. Notice that $\left(\Theta^{(1)}\right)^{1 / 2}(x, y)$ is symmetric in $x$ and $y$ as it should be. Inserting the expression (A.9) for $\left(\Theta^{(1)}\right)^{1 / 2}$ and picking the pole $\omega_{1}=C_{2}(x)$ in the first term and $\omega_{2}=C_{2}(y)$ in the last one, we end up with

$$
\begin{aligned}
\Theta(x, y) & =C_{1}(x) C_{1}(y) \Theta^{(2)}\left(C_{2}(x), C_{2}(y)\right) \\
& =\frac{C_{1}(x) C_{1}(y)}{\sqrt{1-4 \theta^{2}\left(C_{2}(x)\right)^{2}} \sqrt{1-4 \theta^{2}\left(C_{2}(y)\right)^{2}}} \frac{1}{1-\frac{4 \frac{\theta^{2}}{\beta^{2}} C_{2}(x) C_{2}(y)}{\left(1+\sqrt{1-4 \theta^{2}\left(C_{2}(x)\right)^{2}}\right)\left(1+\sqrt{1-4 \theta^{2}\left(C_{2}(y)\right)^{2}}\right)}} .
\end{aligned}
$$

From this expression for the transfer matrix we can read off various facts about the critical properties of our model. First, we see that there are several ways in which the transfer matrix can become singular. One possibility is that the argument of the square root vanishes. Assuming that as usual the critical values 5 of $x$ and $y$ are $x_{c}=y_{c}=1$ this situation occurs when

$$
4 g \theta=1
$$

Another possibility is that the denominator of the second factor in (A.11) vanishes. This corresponds to the situation

$$
2 g \theta\left(\beta+\frac{1}{\beta}\right)=1, \quad \text { and } \quad \beta \leq 1
$$

The critical point (A.13) is the one which reduces to the pure Lorentzian triangulation critical point when $\beta \rightarrow 0$ (more precisely when $h=1$ and $\theta \rightarrow 0$ ). The singularity given by (A.12) corresponds to the critical point of an isolated arch system. As long as $\beta<1$ we always reach the critical point of (A.13) before that of $(\mathrm{A.12})$. For $\beta=1$ the two points coincide and finally for $\beta>1$ only the singularity (A.12) persists. This analysis matches exactly our results in Sect. 4, in particular the relations (4.14), (4.17) and (4.23).

5 The reader should not be surprised that the critical values of $x$ and $y$ differ from those of (5.20). Indeed, the critical values $w_{c}=z_{c}=\frac{1}{2 g}$ of (5.20) correspond to the vanishing of the denominator of $\Theta^{(1)}(w, z)=\oint \frac{d x}{2 i \pi x} \frac{d y}{2 i \pi y}\left(\Theta^{(1)}\right)^{1 / 2}(w, y) \frac{1}{1-x y}\left(\Theta^{(1)}\right)^{1 / 2}(x, z)$, while the denominator of $\left(\Theta^{(1)}\right)^{1 / 2}(x, z)$ vanishes for $x=x_{c}=1$ when $z=z_{c}$, and similarly for $y=y_{c}=1$ when $w=w_{c}$ (by use of $(\mathrm{A} .10)$ ). 
It is obvious that the scaling behavior of $\Theta(x, y)$ in the vicinity of a singularity corresponding to the vanishing of the square root is not compatible with the existence of a Hamiltonian (cf. relation (A.3)). Thus we can only hope to be able to introduce a meaningful continuum time variable as long as $\beta<1$. This conclusion is of course in agreement with the analysis of Sect. 4 . It is, however, not straightforward to write down the Hamiltonian for $\beta<1$ using the relations (A.1) and (A.3), the reason being that for the generalized model we will get not only a pole contribution to the contour integral in (A.3) but also a cut contribution. Introducing the scaling relations

$$
g=\frac{1}{2}\left(1-\frac{1}{2} a^{2} \Lambda\right), \quad x=1-a X, \quad \theta=\left(\beta+\frac{1}{\beta}\right)^{-1},
$$

the contribution from the pole in (A.11) reproduces the the same structure as that of (A.3), up to a redefinition of the renormalized fugacity per triangle, $\Lambda \rightarrow \tilde{\Lambda}=\Lambda\left(\frac{1+\beta^{2}}{1-\beta^{2}}\right)^{2}$. The contribution from the cut is more involved and cannot be obtained so simply. This is not surprising: the Hamiltonian (5.10) (using (5.8)) should read in the language of modified lengths $\tilde{L}=\frac{1}{2} \varphi^{2}=\left(\frac{1-\beta^{2}}{1+\beta^{2}}\right)^{2} L$ :

$$
\hat{H}=-\tilde{L} \frac{\partial^{2}}{\partial \tilde{L}^{2}}-\frac{\partial}{\partial \tilde{L}}+\omega^{2} \tilde{L}+\frac{(\mu-1)^{2}}{4 \tilde{L}} .
$$

When Laplace-transformed in terms of $\tilde{X}$, it becomes

$$
\hat{H} \psi(\tilde{X})=\left(\left(\tilde{X}^{2}-\omega^{2}\right) \frac{\partial}{\partial \tilde{X}}+\tilde{X}\right) \psi(\tilde{X})+\frac{(\mu-1)^{2}}{4} \int_{\tilde{X}}^{\infty} \psi(u) d u
$$

showing that the cut contribution must give rise to a non-local integral term.

Appendix B. The $k$-point function of the scaled numbers of triangles for $\beta<1$

In terms of the time intervals $t_{m}=u_{m+1}-u_{m}$ for $m=1,2, \ldots k-1$, and $t_{k}=$ $T-\sum_{1 \leq m \leq k-1} t_{m}$ the $k$-point correlator of $e^{\mathcal{Z}_{m} \omega \mathcal{N}\left(u_{m}\right)}$ reads

$$
\begin{aligned}
& \left\langle\prod_{m=1}^{k} e^{\mathcal{Z}_{m} \omega \mathcal{N}\left(u_{m}\right)}\right\rangle=\left\langle\prod_{m=1}^{k} e^{\mathcal{Z}_{m} \omega \varphi^{2}\left(u_{m}\right)}\right\rangle \\
& \quad=\frac{1}{Z_{T, \mu}} \int_{0}^{\infty} d \varphi_{1} \ldots d \varphi_{k} \sum_{n_{1}, \ldots, n_{k} \geq 0} \prod_{i=1}^{k} e^{\omega \mathcal{Z}_{i} \varphi_{i}^{2}} \psi_{n_{i}}\left(\varphi_{i}\right) \psi_{n_{i}}\left(\varphi_{i+1}\right) e^{-\omega t_{i}\left(2 n_{i}+\mu\right)},
\end{aligned}
$$


where we have defined $\varphi_{k+1} \equiv \varphi_{1}$. We now use $k$ times the quadratic relation for the modified Laguerre polynomials (5.38) and the change of variables $v_{i}=\omega \varphi_{i}^{2}, i=1,2, \ldots, k$ to rewrite

$$
\left\langle\prod_{m=1}^{k} e^{\mathcal{Z}_{m} \omega \mathcal{N}\left(u_{m}\right)}\right\rangle=2 \sinh (\omega T) e^{\omega T(\mu-1)} \int_{0}^{\infty} \prod_{i=1}^{k} x_{i} d v_{i} \prod_{i=1}^{k} e^{-\delta_{i} v_{i}} I_{\mu-1}\left(2 x_{i} \sqrt{v_{i} v_{i+1}}\right)
$$

where we have set

$$
\begin{aligned}
x_{i} & =\frac{1}{2 \sinh \left(\omega t_{i}\right)}, \quad \delta_{i}=\gamma_{i}-\mathcal{Z}_{i} \\
\gamma_{i} & =2 x_{i} x_{i-1} \sinh \left(\omega\left(t_{i}+t_{i-1}\right)\right)
\end{aligned}
$$

with the convention that $t_{0} \equiv t_{k}$. Let us evaluate by induction the integral

$$
J_{k}\left(x_{1}, \ldots, x_{k} \mid \delta_{1}, \ldots, \delta_{k}\right)=2 \sinh (\omega T) e^{\omega T(\mu-1)} \int_{0}^{\infty} \prod_{i=1}^{k} x_{i} d v_{i} \prod_{i=1}^{k} e^{-\delta_{i} v_{i}} I_{\mu-1}\left(2 x_{i} \sqrt{v_{i} v_{i+1}}\right)
$$

We first perform the integration over $v_{k}$ by applying the following integral relation

$$
r \int_{0}^{\infty} d v e^{-r v} I_{\mu-1}(2 s \sqrt{v}) I_{\mu-1}\left(2 s^{\prime} \sqrt{v}\right)=e^{\frac{1}{r}\left(s^{2}+s^{\prime 2}\right)} I_{\mu-1}\left(\frac{2 s s^{\prime}}{r}\right),
$$

with $r=\delta_{k}, s=x_{k-1} \sqrt{v_{k-1}}$ and $s^{\prime}=x_{k} \sqrt{v_{1}}$, with the result

$$
J_{k}\left(x_{1}, \ldots, x_{k} \mid \delta_{1}, \ldots, \delta_{k}\right)=J_{k-1}\left(x_{1}, x_{2}, \ldots, \frac{x_{k-1} x_{k}}{\delta_{k}} \mid \delta_{1}-\frac{x_{k}^{2}}{\delta_{k}}, \delta_{2}, \ldots, \delta_{k-2}, \delta_{k-1}-\frac{x_{k-1}^{2}}{\delta_{k}}\right) .
$$

This is valid for all $k \geq 3$. When $k=2$, we simply get a single integral

$$
J_{2}\left(x_{1}, x_{2} \mid \delta_{1}, \delta_{2}\right)=\sinh (\omega T) e^{\omega T(\mu-1)} \frac{2 x_{1} x_{2}}{\delta_{2}} \int_{0}^{\infty} d v e^{-\left(\delta_{1}-\frac{x_{1}^{2}+x_{2}^{2}}{\delta_{2}}\right) v} I_{\mu-1}\left(2 v x_{1} x_{2} / \delta_{2}\right),
$$

readily evaluated as a particular case of the Laplace transform of the modified Bessel function (5.45) with $r=\delta_{1}-\frac{x_{1}^{2}+x_{2}^{2}}{\delta_{2}}$ and $s=2 x_{1} x_{2} / \delta_{2}$. This gives

$$
\begin{aligned}
J_{2}\left(x_{1}, x_{2} \mid \delta_{1}, \delta_{2}\right) & =g_{\mu}\left(\frac{2 x_{1} x_{2}}{\delta_{1} \delta_{2}-x_{1}^{2}-x_{2}^{2}}\right) \\
g_{\mu}(s) & =\sinh (\omega T) e^{\omega T(\mu-1)} s^{2-\mu} \frac{\left(1-\sqrt{1-s^{2}}\right)^{\mu-1}}{\sqrt{1-s^{2}}} .
\end{aligned}
$$

Similarly, for $k=1$ we get

$$
\left\langle e^{\mathcal{Z} \omega \mathcal{N}}\right\rangle=f_{\mu}(\mathcal{Z})=J_{1}\left(x_{1} \mid \delta_{1}\right)=g_{\mu}\left(\frac{2 x_{1}}{\delta_{1}}\right)=g_{\mu}\left(\frac{1}{\cosh (\omega T)-\mathcal{Z} \sinh (\omega T)}\right),
$$


In general, applying the recursion relation (B.6), we find

$$
\left\langle\prod_{m=1}^{k} e^{\omega \mathcal{N}\left(u_{m}\right) \mathcal{Z}_{m}}\right\rangle=g_{\mu}\left(\frac{2}{P\left(\mathcal{Z}_{1}, \ldots, \mathcal{Z}_{k} \mid t_{1}, \ldots, t_{k}\right)}\right)
$$

where the polynomial $P$ is defined as follows: we first introduce the sequence

$$
\mu_{k-p}=\delta_{k-p}-\frac{x_{k-p}^{2}}{\mu_{k-p+1}}, \quad p=1,2, \ldots, k-1 \text { with } \quad \mu_{k}=\delta_{k},
$$

and the polynomial $P$ reads

$$
P\left(\mathcal{Z}_{1}, \mathcal{Z}_{2}, \ldots, \mathcal{Z}_{k} \mid t_{1}, t_{2}, \ldots, t_{k}\right)=\frac{\mu_{1} \mu_{2} \ldots \mu_{k}}{x_{1} x_{2} \ldots x_{k}}-\sum_{p=0}^{k-2} \frac{\mu_{2} \mu_{3} \ldots \mu_{k-p}}{x_{1} x_{2} \ldots x_{k-p-1}} \times \frac{x_{k-p} x_{k-p+1} \ldots x_{k}}{\mu_{k-p} \mu_{k-p+1} \ldots \mu_{k}} .
$$

Once expressed in terms of $\mathcal{Z}_{i}$ and $t_{i}$, this polynomial takes the very simple form (5.51), with for instance

$$
\begin{array}{ll}
k=0: & P=2 \cosh (\omega T), \\
k=1: & P\left(\mathcal{Z}_{1} \mid t_{1}=T\right)=2\left(\cosh (\omega T)-\mathcal{Z}_{1} \sinh (\omega T)\right), \\
k=2: & P\left(\mathcal{Z}_{1}, \mathcal{Z}_{2} \mid t_{1}, t_{2}=T-t_{1}\right)=2\left(\cosh (\omega T)-\left(\mathcal{Z}_{1}+\mathcal{Z}_{2}\right) \sinh (\omega T)\right. \\
& \left.+2 \mathcal{Z}_{1} \mathcal{Z}_{2} \sinh \left(\omega t_{1}\right) \sinh \left(\omega t_{2}\right)\right),
\end{array}
$$

leading respectively to the correlators

$$
\begin{aligned}
& k=0:\langle 1\rangle=g_{\mu}\left(\frac{2}{P}\right)=f_{\mu}(0)=1 \\
& k=1:\left\langle e^{\omega \mathcal{N} \mathcal{Z}}\right\rangle=g_{\mu}\left(\frac{2}{P(\mathcal{Z} \mid T)}\right)=f_{\mu}(\mathcal{Z}), \\
& k=2: \quad\left\langle e^{\omega \mathcal{N}\left(u_{1}\right) \mathcal{Z}_{1}} e^{\omega \mathcal{N}\left(u_{2}\right) \mathcal{Z}_{2}}\right\rangle=g_{\mu}\left(\frac{2}{P\left(\mathcal{Z}_{1}, \mathcal{Z}_{2} \mid u_{2}-u_{1}, T+u_{1}-u_{2}\right)}\right) \\
&=f_{\mu}\left(\mathcal{Z}_{1}+\mathcal{Z}_{2}-2 \mathcal{Z}_{1} \mathcal{Z}_{2} \frac{\sinh \left(\omega\left(u_{2}-u_{1}\right)\right) \sinh \left(\omega\left(T+u_{1}-u_{2}\right)\right)}{\sinh (\omega T)}\right),
\end{aligned}
$$

with $f_{\mu}$ as in (5.46). More generally, the $k$-point correlator is expressed as

$$
g_{\mu}\left(s=\frac{2}{P_{k}}\right)=f_{\mu}\left(\operatorname{cotanh}(\omega T)-\frac{P_{k}}{2 \sinh (\omega T)}\right),
$$

in terms of $P_{k} \equiv P\left(\mathcal{Z}_{1}, \ldots, \mathcal{Z}_{k} \mid t_{1}, \ldots, t_{k}\right)$.

As a final check on the result (5.51), we see that

$$
\begin{aligned}
P\left(\mathcal{Z}_{1}, \ldots, \mathcal{Z}_{k-1}, \mathcal{Z}_{k}=0 \mid t_{1}, \ldots, t_{k}\right) & =P\left(\mathcal{Z}_{1}, \ldots, \mathcal{Z}_{k-1} \mid t_{1}, \ldots, t_{k-1}\right) \\
P\left(\mathcal{Z}_{1}, \ldots, \mathcal{Z}_{k} \mid t_{1}, \ldots, t_{k-1}, t_{k}=0\right) & =P\left(\mathcal{Z}_{1}, \ldots, \mathcal{Z}_{k-2}, \mathcal{Z}_{k-1}+\mathcal{Z}_{k} \mid t_{1}, \ldots, t_{k-1}\right),
\end{aligned}
$$

as expected from the definition of the correlator. 


\section{Appendix C. Comparison with the harmonic oscillator}

We wish to compute the $k$-point correlation function of the operator $e^{\omega \varphi^{2}(u) \mathcal{Z}}$ in the one-dimensional quantum system with Hamiltonian

$$
H_{\mathrm{osc}}=-\frac{1}{2} \frac{d^{2}}{d \varphi^{2}}+\frac{1}{2} \omega^{2} \varphi^{2}
$$

on a time circle of length $T$. The Hamiltonian has eigenvalues $E_{n}=\omega\left(n+\frac{1}{2}\right)$ and eigenfunctions

$$
\psi_{n}^{\mathrm{osc}}(\varphi)=\sqrt{\frac{\sqrt{\omega}}{\sqrt{\pi} 2^{n} n !}} H_{n}(\sqrt{\omega} \varphi) e^{-\frac{1}{2} \omega \varphi^{2}},
$$

where $H_{n}(x)$ are the Hermite polynomials, $n=0,1,2, \ldots$ and $\varphi \in(-\infty,+\infty)$. Next we will use the following quadratic relation obeyed by the Hermite polynomials

$$
\sum_{n=0}^{\infty} H_{n}(x) H_{n}(y) \frac{(z / 2)^{n}}{n !}=\frac{1}{\sqrt{1-z^{2}}} e^{\frac{2 x y z-z^{2}\left(x^{2}+y^{2}\right)}{1-z^{2}}},
$$

immediately translated into

$$
\sum_{n \geq 0} \psi_{n}^{\mathrm{osc}}\left(\varphi_{0}\right) \psi_{n}^{\mathrm{osc}}\left(\varphi_{1}\right) z^{n+\frac{1}{2}}=\sqrt{\frac{\omega z}{\pi\left(1-z^{2}\right)}} e^{\frac{2 z}{1-z^{2}} \omega \varphi_{0} \varphi_{1}-\frac{1+z^{2}}{1-z^{2}} \frac{\omega}{2}\left(\varphi_{0}^{2}+\varphi_{1}^{2}\right)}
$$

The correlation function reads

$$
\begin{aligned}
& \left\langle\prod_{m=1}^{k} e^{\omega \varphi^{2}\left(u_{m}\right) \mathcal{Z}_{m}}\right\rangle_{\mathrm{osc}} \\
& \quad=\frac{1}{Z_{T, \mathrm{osc}}} \int_{\mathbb{R}} d \varphi_{1} \ldots d \varphi_{k} \sum_{n_{1}, \ldots, n_{k} \geq 0} \prod_{i=1}^{k} e^{-\omega t_{i}\left(n_{i}+\frac{1}{2}\right)} \psi_{n_{i}}^{\mathrm{osc}}\left(\varphi_{i}\right) \psi_{n_{i}}^{\mathrm{osc}}\left(\varphi_{i+1}\right) e^{\mathcal{Z}_{i} \omega \varphi_{i}^{2}},
\end{aligned}
$$

where the time intervals $t_{i}=u_{i+1}-u_{i}, i=1,2, \ldots, k-1, t_{k}=T-\sum_{1 \leq i \leq k-1} t_{i}$. Applying $k$ times the relation (C.4) with respectively $\varphi_{0}, \varphi_{1} \rightarrow \varphi_{i}, \varphi_{i+1}$ and $z \rightarrow e^{-\omega t_{i}}$, and performing the change of variables $v_{i}=\sqrt{2 \omega} \varphi_{i}$, we get

$$
\left\langle\prod_{m=1}^{k} e^{\omega \varphi^{2}\left(u_{m}\right) \mathcal{Z}_{m}}\right\rangle_{\mathrm{osc}}=\frac{\sqrt{x_{1} \ldots x_{k}}}{Z_{T, \mathrm{osc}}} \int_{\mathbb{R}}\left(\prod_{i=1}^{k} \frac{d v_{i}}{\sqrt{2 \pi}}\right) e^{-\frac{1}{2} \sum_{i=1}^{k} \delta_{i} v_{i}^{2}-2 x_{i} v_{i} v_{i+1}},
$$

with $x_{i}$ and $\delta_{i}$ as in (B.3). This finally yields

$$
\left\langle\prod_{m=1}^{k} e^{\omega \varphi^{2}\left(u_{m}\right) \mathcal{Z}_{m}}\right\rangle_{\text {osc }}=2 \sinh (\omega T / 2) \sqrt{x_{1} \ldots x_{k}} \operatorname{det}(M-Z)^{-\frac{1}{2}}=\sqrt{\frac{\operatorname{det}(M)}{\operatorname{det}(M-Z)}},
$$

as a straightforward result of the Gaussian integration, with $M$ and $Z$ as in (5.57). The comparison with the alternative expression (5.55) immediately yields the determinantal expression for the polynomial $P$ (5.56). 


\section{References}

[1] J. Ambjørn, B. Durhuus and T. Johnsson, Quantum Geometry, Cambridge University Press, 1997.

[2] F. David, Nucl. Phys. B257 (1985) 45; V.A. Kazakov, Phys. Lett. 150B (1986) 140.

[3] J. Ambjørn and R. Loll, Nucl. Phys. B536 (1998) 407, hep-th/9805108.

[4] R. Nakayama, Phys. Lett. B 325 (1994) 347.

[5] B. Duplantier and I. Kostov, Phys. Rev. Lett. 61 (1988) 559.

[6] J. Ambjørn, J. Correia, C. Kristjansen and R. Loll, Phys. Lett. B475 (2000) 24, hepth/9912267.

[7] B. Duplantier and I. Kostov, Nucl. Phys. B340 (1990) 491; B. Eynard, E. Guitter and C. Kristjansen, Nucl. Phys. B528 (1998) 523, cond-mat/9801281; S. Higuchi, Mod. Phys. Lett. A 13 (1998) 727, cond-mat/9806349.

[8] J. Ambjørn, K.N. Anagnostopoulos and R. Loll, Phys. Rev. D60 (1999) 104035, hepth/9904012; Phys. Rev. D61 (2000) 044010, hep-lat/9909129.

[9] P. Di Francesco, E. Guitter and C. Kristjansen, Nucl. Phys. B567 (2000) 515, hepth/9907084.

[10] V.G. Knizhnik, A.M. Polyakov and A.B. Zamolodchikov, Mod. Phys. Lett. A3 (1988) 819.

[11] L.D. Landau and E.M. Lifshitz, Quantum Mechanics, Pergamon Press, 1958.

[12] H. Bateman, Higher Transcendental Functions, Vol. I, McGraw-Hill (1953).

[13] H. Bateman, Higher Transcendental Functions, Vol. II, McGraw-Hill (1953).

[14] C. Itzykson and J.-M. Drouffe, Statistical Field Theory, Cambridge University Press 1989.

[15] H. Spohn, Fixed points of a functional renormalization group for critical wetting, Europhys. Lett. 14 (1991) 689. 